\title{
SOME STABILITY RESULTS UNDER DOMAIN VARIATION FOR NEUMANN PROBLEMS IN METRIC SPACES
}

\author{
Estibalitz Durand-Cartagena and Antoine Lemenant \\ Universidad Complutense de Madrid, Departamento de Análisis Matemático \\ 28040 Madrid, Spain; estibalitzdurand@mat.ucm.es \\ Scuola Normale Superiore, Centro E. De Giorgi \\ Piazza dei Cavalieri 3, I-56100 Pisa, Italy; antoine.lemenant@sns.it
}

\begin{abstract}
A famous result of Chenais [8] (1975) says that if $\Omega_{n}$ is a sequence of extension domains in $\mathbf{R}^{N}$ that converges to $\Omega$ in the characteristic functions topology, then the weak solutions $u_{n}$ for the problem

$$
\begin{cases}-\Delta u_{n}+u_{n}=f & \text { in } \Omega_{n} \\ \frac{\partial}{\partial \nu} u_{n}=0 & \text { on } \partial \Omega_{n}\end{cases}
$$

converge strongly to the solution $u$ of the same problem in $\Omega$. It is also proved in [8] using the method of Calderón that an $\varepsilon$-cone condition is sufficient to obtain uniform extension domains. In this paper we establish this result in a metric space framework, replacing the classical Sobolev space $H^{1}(\Omega)$ by the Newtonian space $N^{1,2}(\Omega)$. Moreover, using the latest results about extension domains contained in [2], and which rely on the techniques of Jones, we give weaker conditions on the domains for still getting stability of the Neumann problem. Finally we prove that the Neumann problem is stable for a sequence of quasiballs with uniform distortion constant that converge in a certain measure sense. The latter result gives a new existence theorem for some shape optimisation problems under quasiconformal variations.
\end{abstract}

\section{Introduction}

In this paper we focus on the following question. If $\Omega_{n}$ is a sequence of domains that converges to $\Omega$ (in a certain sense), is it true that the solutions $u_{n}$ of the Neumann problem

$$
\begin{cases}-\Delta u_{n}+u_{n}=f & \text { in } \Omega_{n}, \\ \frac{\partial}{\partial \nu} u_{n}=0 & \text { on } \partial \Omega_{n}\end{cases}
$$

converge to the solution $u$ of the same Neumann problem in $\Omega$ ? This question, related to shape optimisation problems and domain identification problems, was studied in the past for both Dirichlet and Neumann boundary conditions. The Dirichlet problem was in particular investigated a lot and some results involving capacity conditions are close to be optimal (see for instance [5]). On the other hand, the Neumann problem seems more difficult and very little has been done in dimension greater than 2 (see $[8,6,10,18]$ and the references therein) and the classical "Neumann sieve" (see $[11,26])$ shows that in general the convergence cannot be true without topological constraints on the sequence of domains.

doi:10.5186/aasfm.2010.3533

2000 Mathematics Subject Classification: Primary 58J99, 30L10, 49J99, 46E35.

Key words: Newtonian spaces, $\gamma$-convergence, Mosco convergence, Neumann problem, differentiability in metric spaces, quasiconformal mappings, shape optimisation. 
In a famous paper of Chenais [8] (1975), it is shown that if $\Omega_{n} \subset \mathbf{R}^{N}$ is a sequence of extension domains that converge to $\Omega$ in the characteristic functions topology, then the weak solutions $u_{n}$ for the problem (0.2) converge strongly to the solution $u$ of the same problem in $\Omega$, more precisely, $\chi_{\Omega_{n}} u_{n}$ strongly converges to $\chi_{\Omega} u$ in $L^{2}\left(\mathbf{R}^{N}\right)$ and $\chi_{\Omega_{n}} \nabla u_{n}$ strongly converges to $\chi_{\Omega} \nabla u$ in $L^{2}\left(\Omega, \mathbf{R}^{N}\right)$. It is also proved in [8] using the method of Calderón that a uniform $\varepsilon$-cone condition is sufficient to obtain extension domains. It is worth mentioning that this condition implies a Lipschitz regularity on the boundary of the domain.

In the first part of the present paper (Sections 1 to 4 ) we extend the result of Chenais in two different directions. First, we place ourselves into a metric space framework replacing the classical Sobolev space $H^{1}(\Omega)$ by the Newtonian space $N^{1,2}(\Omega)$ (Section 1). In Section 2 we give a possible definition of Problem (0.2) in this setting while in Section 3 we prove that, as for the Euclidean case, the stability is equivalent to a convergence of Newtonian spaces in the sense of Mosco.

Then we improve in Section 4 the $\varepsilon$-cone condition involving the latest results about extension domains contained in [2]. Indeed, in [19], Jones introduced a class of domains called $(\varepsilon, \delta)$-flat domains, that are in particular weaker than Lipschitz domains, and he proved that they are extension domains. This result was then extended to different contexts in the last 20 years (see [2] and the references therein) and the particular case of Newtonian spaces was considered in [2] with sophisticated geometrical conditions on the domains. This allows us to state some new stability results under geometric conditions on the boundaries of the domains (see Theorem 23).

In the second part of the paper (Section 5) we prove a new stability result for a larger class of domains than the class of extension domains. Indeed, we prove that the stability holds along a sequence of quasiconformal perturbations of a fixed domain, that converges in the Hausdorff metric and in a certain measure sense (see Theorem 33). This stability result, proved here in a general metric space framework, is interesting even for the Euclidean case. It also implies an existence theorem for a class of shape optimisation problems under quasiconformal variations with Neumann boundary condition (Theorem 35).

Acknowledgements. The authors wish to warmly thank Nages Shanmugalingam for many interesting discussions on this subject and helpful remarks about the redaction of this paper. They also express their gratitude to the anonymous referee who carefully read the manuscript and suggested many helpful corrections. The first author is supported in part by DGES (Spain) Project MTM2006-03531 and by the Grant AP2006-00620.

\section{Notation and preliminaries}

We always assume that $(X, d, \mu)$ is a metric measure space, where $\mu$ is a Borel regular measure, that is, $\mu$ is an outer measure on a metric space $(X, d)$ such that all Borel sets are $\mu$-measurable and for each set $A \subset X$ there exists a Borel set $B$ such that $A \subset B$ and $\mu(A)=\mu(B)$.

Definition 1. We say that a measure $\mu$ on $X$ is doubling if there is a positive constant $C_{\mu}$ such that

$$
0<\mu(B(x, 2 r)) \leq C_{\mu} \mu(B(x, r))<\infty
$$


for each $x \in X$ and $r>0$. Here $B(x, r)$ denotes the open ball with center $x$ and radius $r>0$.

Whenever we work in the Euclidean setting, that is $X:=\mathbf{R}^{N}$, we will assume $\mu$ to be the $N$-dimensional Lebesgue measure which will be denoted by $\mathscr{L}^{N}$. Observe that $\mathscr{L}^{N}$ is always a doubling measure with any choice of distance coming from a norm on $\mathbf{R}^{N}$.

We say that $(X, d, \mu)$ is a $Q$-regular space if there exists a constant $C_{0}$ such that for every ball $B(x, r)$ contained in $X$,

$$
C_{0}^{-1} r^{Q} \leq \mu(B(x, r)) \leq C_{0} r^{Q} .
$$

In the following we will place ourselves in the context of Sobolev spaces defined in metric measure spaces. The reader is encouraged to consult the overview article [13] by Hajłasz for an introduction to Sobolev spaces in metric measure spaces. It should be pointed out here, that if the space supports a $p$-Poincaré inequality, $1<p<\infty$ (see Definition 3), all the approaches to Sobolev spaces described in [13] are equivalent (see Theorem 1.0.6 in [20]).

For the record, we recall here the definition of Newtonian Spaces, introduced by Shanmugalingam [27]. Its definition is based on the notion of $p$-weak upper gradients.

Definition 2. Let $p \geq 1$. A non-negative Borel function $g$ on $X$ is a $p$-weak upper gradient of an extended real-valued function $u$ on $\mathrm{X}$, if it holds

$$
|u(\gamma(a))-u(\gamma(b))| \leq \int_{\gamma} g
$$

except for a curve family with zero $p$-modulus (see for example [16] for the definition of the $p$-modulus of a curve family). By a curve $\gamma$ we mean a continuous mapping $\gamma:[a, b] \rightarrow X$. The image of a curve will be denoted by $|\gamma|=\gamma([a, b])$.

Among the set of all $p$-weak upper gradients of a function $u$, there is a smallest member in the $L^{p}$-norm, called the minimal weak upper gradient. The minimal weak upper gradient $\rho_{u}$ is unique up to a set of measure zero and $\rho_{u} \leq g$ a.e. for all $p$-weak upper gradients $g \in L_{\text {loc }}^{p}(X)$ of $u$.

Definition 3. Let $1 \leq p<\infty$. We say that $(X, d, \mu)$ supports a weak $(1, p)$ Poincaré inequality if there exist constants $C_{p}>0$ and $\lambda \geq 1$ such that for every Borel measurable function $u: X \rightarrow \mathbf{R}$ and every upper gradient $g: X \rightarrow[0, \infty]$ of $u$, the pair $(u, g)$ satisfies the inequality

$$
f_{B(x, r)}\left|u-u_{B(x, r)}\right| d \mu \leq C_{p} r\left(f_{B(x, \lambda r)} g^{p} d \mu\right)^{1 / p},
$$

for each $B(x, r) \subset X$.

Here for arbitrary $A \subset X$ with $0<\mu(A)<\infty$ we write

$$
u_{A}=f_{A} u=\frac{1}{\mu(A)} \int_{A} u d \mu \text {. }
$$

If the space is $Q$-regular, then a $(1, p)$-Poincaré inequality implies the a priori stronger inequality where one replaces (for all balls) the averaged $L^{1}$-norm on the left by the averaged $L^{q}$ - norm for some $q>p$; we could so speak about $(q, p)$-Poincaré inequalities. 
Theorem 4. [14, Theorem 1] Suppose that $X$ is $Q$-regular and that $(u, g)$ satisfies a $(1, p)$-Poincaré inequality for some $1 \leq p \leq Q$. Then $(u, g)$ satisfies a weak $(q, p)$-Poincaré inequality for $1 \leq q<p Q /(Q-p)$. In the special case $p=Q$, the right-hand term is the $L^{\infty}$-norm of $g$.

In the special case $q=1$ we simply write weak $p$-Poincaré inequality.

The Poincaré inequality creates a link between the measure, the metric and the gradient and it provides a way to pass from the infinitesimal information which gives the gradient to larger scales. Metric spaces with doubling measure and Poincaré inequality admit first order differential calculus akin to that in Euclidean spaces.

Let $\widetilde{N}^{1, p}(X, d, \mu)$, where $1 \leq p<\infty$, be the class of all $L^{p}$ integrable Borel functions on $X$ for which there exists a $p$-weak upper gradient in $L^{p}$. For $u \in$ $\widetilde{N}^{1, p}(X, d, \mu)$ we define

$$
\|u\|_{\widetilde{N}^{1, p}}=\|u\|_{L^{p}}+\inf _{g}\|g\|_{L^{p}},
$$

where the infimum is taken over all $p$-weak upper gradients $g$ of $u$. Now, we define in $\widetilde{N}^{1, p}$ an equivalence relation by $u \sim v$ if and only if $\|u-v\|_{\widetilde{N}^{1, p}}=0$.

Definition 5. The Newtonian space $N^{1, p}(X, d, \mu)$ is defined as the quotient $\widetilde{N}^{1, p}(X, d, \mu) / \sim$ and it is equipped with the norm $\|u\|_{N^{1, p}}=\|u\|_{\widetilde{N}^{1, p}}$.

Let us mention that the space $\left(N^{1, p}(X),\|\cdot\|_{N^{1, p}}\right)$ is a Banach space (see Theorem 3.7 in [27]). In the sequel we will need the following technical Lemma.

Lemma 6. [27, 4.11] Let $p>1$ and let $\left\{u_{k}\right\}_{k \geq 0}$ be a sequence of functions in $L^{p}(X)$ with upper gradients $\left\{g_{k}\right\}_{k \geq 0}$ in $L^{p}(X)$ such that $u_{k}$ weakly converges to $u$ in $L^{p}$ and $g_{k}$ weakly converges to $g$ in $L^{p}$. Then $g$ is a $p$-weak upper gradient for $u$, after modifying $u$ on a set of measure zero.

Now, we focus our attention in the exponent $p=2$. In this case, the energy integral,

$$
\mathscr{E}_{1}(u)=\int_{X} \rho_{u}{ }^{2} d \mu, \quad u \in N^{1,2}(X)
$$

defines a Dirichlet energy form (for more information about Dirichlet forms in the context of metric measure spaces we refer to the reader to [22]). This energy form is a priori not bilinear, since the pallelogram rule is not known to hold in general. Namely, the validity of the equality $\rho_{u+v}{ }^{2}+\rho_{u-v}{ }^{2}=2\left(\rho_{u}{ }^{2}+\rho_{v}{ }^{2}\right)$ is not clear. However, in this case an equivalent bilinear Dirichlet form on $N^{1,2}(X)$ can be constructed using Cheeger's differentiation theory. Cheeger in [7] gave an alternative definition of Sobolev spaces which leads to the same space (see [27, 4.10]). Cheeger's definition yields the notion of partial derivatives in the following theorem.

Theorem 7. [7, 4.38] Let $X$ be a metric space that supports a doubling Borel measure $\mu$ which is non-trivial and finite on balls and suppose that $X$ supports a weak $p$-Poincaré inequality for some $1 \leq p<\infty$. Then there exists a countable collection $\left(X_{\alpha}, \mathbf{x}_{\alpha}\right)$ of measurable sets $X_{\alpha} \subset X$ and Lipschitz coordinates

$$
\mathbf{x}_{\alpha}=\left(x_{\alpha}^{1}, \ldots, x_{\alpha}^{N(\alpha)}\right): X \longrightarrow \mathbf{R}^{N(\alpha)} \quad \text { where } \quad 0 \leq N(\alpha)<\infty
$$

such that:

(1) $X=\bigcup_{\alpha} X_{\alpha}$ and $\mu\left(X \backslash \bigcup_{\alpha} X_{\alpha}\right)=0$. 
(2) There exists $N \geq 0$ such that $N(\alpha) \leq N$ for each $\left(X_{\alpha}, \mathbf{x}_{\alpha}\right)$.

(3) If $u: X \rightarrow \mathbf{R}$ is Lipschitz, then there exists a unique measurable bounded vector valued function such that $d u^{\alpha}: X_{\alpha} \longrightarrow \mathbf{R}^{N(\alpha)}$

$$
\lim _{\substack{y \rightarrow x \\ y \neq x}} \frac{\left|u(y)-u(x)-d u^{\alpha}(x) \cdot\left(\mathbf{x}_{\alpha}(y)-\mathbf{x}_{\alpha}(x)\right)\right|}{d(y, x)}=0
$$

for $\mu$-a.e. $x \in X_{\alpha}$.

We can assume that the sets $X_{\alpha}$ are pairwise disjoint and extend $d u^{\alpha}$ by zero outside $X_{\alpha}$. Now we put $D u=\sum_{\alpha} d u^{\alpha}$, regarding $d u^{\alpha}(x)$ as vectors in $\mathbf{R}^{N}$ and the differential mapping $D: u \rightarrow D u$ is linear.

The previous theorem establishes a version of Rademacher's theorem for Lipschitz functions in the context of metric measure spaces. Cheeger constructs a finitedimensional vector bundle $\mathbf{F}$ over $X$ (the generalized cotangent bundle) and the differential operator $D$ which takes $N^{1,2}(X)$ into the $L^{2}$-sections of $\mathbf{F}$. The pointwise norms $\|\cdot\|_{x}$ on the fibers of $\mathbf{F}$ is defined so that the identity $\|D u(x)\|_{x}=\rho_{u}(x)$ holds almost everywhere. Thus the Dirichlet form of (1.2) can be rewritten

$$
\mathscr{E}_{2}(u)=\int_{X}\|D u\|^{2}
$$

Since the fibers of $\mathbf{F}$ are uniformly finite-dimensional, they can be uniformly renormed with equivalent inner product norms $\||\cdot|\|_{x}=\langle\cdot, \cdot\rangle_{x}^{1 / 2}$, so that for all $u \in N^{1,2}(X)$ we have $\||D u|\|_{x} \simeq \rho_{u}(x)$ for $\mu$-almost every $x \in X$. Then,

$$
\mathscr{E}(u)=\int_{X}\||D u|\|^{2}, \quad u \in N^{1,2}(X)
$$

defines a new Dirichlet form which is equivalent to (1.3) on $N^{1,2}(X)$. The usual polarization technique yields the bilinear form

$$
E(u, v)=\int\langle D u, D v\rangle d \mu .
$$

In that way, $N^{1,2}(X)$ becomes a Hilbert space with the inner product

$$
\langle u, v\rangle_{N^{1,2}(\Omega)}:=\int_{X} D u \cdot D v d \mu+\int_{X} u v d \mu .
$$

The following Lemma will be also useful in the sequel.

Lemma 8. Let $(X, \mu)$ be a measurable space and let $u_{n}: X \rightarrow \mathbf{R}, \phi_{n}: X \rightarrow \mathbf{R}$ be two sequences of measurable functions such that $u_{n}$ is uniformly bounded in $L^{2}(X, \mu)$, $u_{n}$ weakly converges to $u$, and $\phi_{n}$ strongly converges to $\phi$ in $L^{2}(X, \mu)$. Then

$$
\int_{X} u_{n}(x) \phi_{n}(x) d \mu(x) \rightarrow \int_{X} u(x) \phi(x) d \mu(x) .
$$

Proof. We have that

$$
\int_{X} u_{n} \phi_{n} d \mu=\int_{X}\left(u_{n}-u\right) \phi d \mu+\int_{X}\left(u_{n}-u\right)\left(\phi_{n}-\phi\right) d \mu+\int_{X} u \phi_{n} d \mu .
$$

By the weak convergence of $u_{n}$ we know that $\int_{X}\left(u_{n}-u\right) \phi d \mu \rightarrow 0$ and by the strong convergence of $\phi_{n}$ we also have $\int_{X} u \phi_{n} d \mu \rightarrow \int_{X} u \phi d \mu$. So it is enough to prove that

$$
\int_{X}\left(u_{n}-u\right)\left(\phi_{n}-\phi\right) d \mu \rightarrow 0 \text {. }
$$


But since $u_{n}$ is uniformly bounded in $L^{2}(X)$ we have that

$$
\int_{X}\left(u_{n}-u\right)\left(\phi_{n}-\phi\right) d \mu \leq\left\|u_{n}-u\right\|_{L^{2}}\left\|\phi_{n}-\phi\right\|_{L^{2}} \leq C\left\|\phi_{n}-\phi\right\|_{L^{2}}
$$

and we conclude using the strong convergence of $\phi_{n}$.

Throughout this paper constants are labeled $C$, and the value of $C$ might change even from line to line.

\section{The Neumann problem on metric spaces}

In this section we want to give a weak sense to the equation

$$
\begin{cases}-\Delta u+u=f & \text { in } \Omega, \\ \frac{\partial u}{\partial \nu}=0 & \text { on } \partial \Omega\end{cases}
$$

in a general metric space framework. In [1] a general Dirichlet problem for $p$-harmonic functions has already been studied under the conditions that the measure on the space is doubling and supports a Poincaré inequality. In the wake of [1], we will use the next definition.

Definition 9. Let $\Omega$ be an open subspace of $X$ and let $L$ be a bounded linear form on $L^{2}(\Omega) \times L^{2}\left(\Omega, \mathbf{R}^{N}\right)$. A function $u: X \rightarrow[-\infty, \infty]$ is said to solve the Neumann problem associated to $L$ if $u \in N^{1,2}(\Omega)$ and for all $\phi \in N^{1,2}(\Omega)$,

$$
\int_{\Omega} D u \cdot D \phi d \mu+\int_{\Omega} u \phi d \mu=\bar{L}(\phi)
$$

where $\bar{L}: \phi \mapsto L(\phi, D \phi)$.

Actually Problem (2.1) corresponds to the case $\bar{L}(\phi):=\int_{\Omega} \phi f$. Observe that when $L$ is a bounded linear form on $L^{2}(\Omega) \times L^{2}\left(\Omega, \mathbf{R}^{N}\right)$, then it induces a bounded linear form on $N^{1,2}(\Omega)$. Since $N^{1,2}(\Omega)$ is a Hilbert space endowed with the scalar product

$$
\langle u, v\rangle_{N^{1,2}(\Omega)}:=\int_{\Omega} D u \cdot D v d \mu+\int_{\Omega} u v d \mu,
$$

we deduce by Lax-Milgram Theorem that for any bounded linear form $L$ on $L^{2}(\Omega) \times$ $L^{2}\left(\Omega, \mathbf{R}^{N}\right)$ there exists a unique solution $u \in N^{1,2}(\Omega)$ satisfying equation $(*)$. This solution can be obtained by minimizing the energy

$$
J(v):=\frac{1}{2}\langle v, v\rangle_{N^{1,2}(\Omega)}-\bar{L}(v) .
$$

For every $L$ and $\Omega$ we will denote by $u_{\Omega}^{L}$ this solution. For the special case when $\bar{L}(u)=\int_{\Omega} u f$ we will also denote $u_{\Omega}^{f}$.

Applying (*) with $\phi=u_{\Omega}^{L}$ we get the identity

$$
\left\|u_{\Omega}^{L}\right\|_{N^{1,2}(\Omega)}^{2} \simeq\left(\int_{\Omega}\left|D u_{\Omega}^{L}\right|^{2} d \mu+\int_{\Omega}\left|u_{\Omega}^{L}\right|^{2} d \mu\right)=\bar{L}\left(u_{\Omega}^{L}\right)
$$

which implies in particular

$$
\left\|u_{\Omega}^{L}\right\|_{N^{1,2}(\Omega)} \leq C\|L\|,
$$

where $\|L\|$ is the norm of $L$ as a bounded operator on $L^{2}(\Omega) \times L^{2}\left(\Omega, \mathbf{R}^{N}\right)$. 


\section{Mosco-convergence and $\gamma$-convergence}

In this section, we will study the relationship between stability for the Neumann problem described in the previous section and Mosco-convergence, a classical notion of convergence introduced by Mosco [25] related to variational problems and very close to $\Gamma$-convergence for functionals. We refer to $[9,3,18]$ for some surveys about the subject.

Let $\Omega$ be a domain of $X$ and $p \geq 1$. For every $u \in N^{1, p}(\Omega)$ we denote

$$
\hat{u}:=\left(u \chi_{\Omega}, D u \chi_{\Omega}\right) \in L^{p}(X) \times L^{p}\left(X, \mathbf{R}^{N}\right),
$$

and we introduce the closed linear subspace $\mathscr{B}_{\Omega}^{p}$ of $L^{p}(X) \times L^{p}\left(X, \mathbf{R}^{N}\right)$,

$$
\mathscr{B}_{\Omega}^{p}:=\left\{\hat{u} ; u \in N^{1, p}(\Omega)\right\} .
$$

When $p=2$ we will simply denote $\mathscr{B}_{\Omega}$. Recall here that by the work of Franchi, Hajłasz and Koskela $([12])$, it follows that $\mathscr{B}_{\Omega}^{p}$ is a closed subspace of the Banach space $L^{p}(X) \times L^{p}\left(X, \mathbf{R}^{N}\right)$.

Definition 10. Let $\left\{\Omega_{n}\right\}_{n \in \mathbf{N}}$ and $\Omega$ be some domains contained in $X$. We say that the sequence $\Omega_{n} \gamma$-converges to $\Omega$ and we denote

$$
\Omega_{n} \stackrel{\gamma}{\rightarrow} \Omega
$$

if for every bounded linear form $L$ on $L^{2}(X) \times L^{2}\left(X, \mathbf{R}^{N}\right)$ we have that $\hat{u}_{\Omega_{n}}^{L}$ strongly converges in $L^{2}(X) \times L^{2}\left(X, \mathbf{R}^{N}\right)$ to $\hat{u}_{\Omega}^{L}$.

Remark 11. To be coherent with Definition 9, when considering $u_{\Omega_{n}}^{L}$, the linear form $L$ has to be understood as the canonical linear form on $L^{2}\left(\Omega_{n}\right) \times L^{2}\left(\Omega_{n}, \mathbf{R}^{N}\right)$ induced by the given linear form on $L^{2}(X) \times L^{2}\left(X, \mathbf{R}^{N}\right)$ by the formula

$$
L(u, v)=L\left(\chi_{\Omega_{n}} u, \chi_{\Omega_{n}} v\right)
$$

where $(u, v)$ lies in $L^{2}\left(\Omega_{n}\right) \times L^{2}\left(\Omega_{n}, \mathbf{R}^{N}\right)$. Sometimes in the proofs, the characteristic function $\chi_{\Omega_{n}}$ will be omitted.

If $\Omega_{n} \gamma$-converges to $\Omega$ we will also say that the Neumann problem is stable. It is well known that in the Euclidean case, $\gamma$-convergence of $\Omega_{n}$ is equivalent to Mosco-convergence of $\mathscr{B}_{\Omega_{n}}$.

Definition 12. (Mosco-convergence) Let $\Omega_{k}$ and $\Omega$ be open subsets of $X$ and let $\mathscr{B}_{\Omega_{k}}^{p}$ and $\mathscr{B}_{\Omega}^{p}$ be the corresponding subspaces of $L^{p}(X) \times L^{p}\left(X, \mathbf{R}^{N}\right)$. We say that $\mathscr{B}_{\Omega_{k}}^{p}$ converges to $\mathscr{B}_{\Omega}^{p}$ in the sense of Mosco if the following two properties hold:

(M1) For every $u \in N^{1, p}(\Omega)$, there exists a sequence $u_{k} \in N^{1, p}\left(\Omega_{k}\right)$ such that $u_{k} \chi_{\Omega_{k}}$ converges to $u \chi_{\Omega}$ strongly in $L^{p}(X)$ and $D u_{k} \chi_{\Omega_{k}}$ converges to $D u \chi_{\Omega}$ strongly in $L^{p}\left(X, \mathbf{R}^{N}\right)$.

(M2) If $h_{k}$ is a sequence of indices converging to $\infty, u_{h_{k}}$ is a sequence such that $u_{h_{k}} \in N^{1, p}\left(\Omega_{h_{k}}\right)$ for every $k$, and $u_{h_{k}} \chi_{\Omega_{h_{k}}}$ converges weakly in $L^{p}(X)$ to a function $\phi$, while $D u_{h_{k}} \chi_{\Omega_{h_{k}}}$ converges weakly in $L^{p}\left(X, \mathbf{R}^{N}\right)$ to a function $\psi$, then there exists $u \in N^{1, p}(\Omega)$ such that $\phi=u \chi_{\Omega}$ and $\psi=D u \chi_{\Omega} \mu$-a.e. in $X$.

As we shall see in the following, the link between Mosco convergence and $\gamma$ convergence still holds in metric spaces. The proof relies on the same argument that the standard proof in $\mathbf{R}^{N}$ (see for instance Proposition 3.7.10 in [18]) and we write here the full details for the convenience of the reader. 
Proposition 13. Let $\Omega_{n} \subseteq X$ where $(X, d, \mu)$ is a metric measure space such that the embedding $N^{1,2}(X) \hookrightarrow L^{2}(X)$ is compact. Then $\Omega_{n} \gamma$-converges to $\Omega$ if and only if $\mathscr{B}_{\Omega_{n}}$ converges to $\mathscr{B}_{\Omega}$ in the sense of Mosco.

Proof. First, let us prove that Mosco-convergence implies $\gamma$-convergence. Indeed, assume that $\mathscr{B}_{\Omega_{n}}$ Mosco-converges to $\mathscr{B}_{\Omega}$ and let $u_{n}:=u_{\Omega_{n}}^{L}$ be a sequence of solutions for equation $(*)$ associated to a given linear form $L$. By $(2.2)$ we know that the sequence $\hat{u}_{n}$ is uniformly bounded in $L^{2}(X) \times L^{2}\left(X, \mathbf{R}^{N}\right)$ and so, we can extract a subsequence $\hat{u}_{n_{k}}$ of $\hat{u}_{n}$ such that $u_{n_{k}}$ weakly converges to a function $\varphi$ and $D u_{n_{k}}$ weakly converges to another function $\psi$. Now, Mosco-convergence (condition (M2)) implies that $(\varphi, \psi) \in \mathscr{B}_{\Omega}$. In other words there exists $u \in N^{1,2}(\Omega)$ such that $(\varphi, \psi)=$ $\hat{u}$.

Now for all $\phi \in N^{1,2}(\Omega)$, by property (M1) we know that there exists a sequence of functions $\phi_{n} \in N^{1,2}\left(\Omega_{n}\right)$ such that $\hat{\phi}_{n}$ strongly converges to $\hat{\phi}$ in $L^{2}(X) \times L^{2}\left(X, \mathbf{R}^{N}\right)$. Then, applying Lemma 8 we can pass to the limit, strongly in $\phi_{n_{k}}$ and weakly in $u_{n_{k}}$ in the following identity

$$
\int_{X} D u_{n_{k}} \cdot D \phi_{n_{k}} d \mu+\int_{X} u_{n_{k}} \phi_{n_{k}} d \mu=L\left(\phi_{n_{k}}, D \phi_{n_{k}}\right)
$$

to obtain that the function $u=u_{\Omega}^{L}$, is the solution of $(*)$ in $\Omega$. Moreover, since $L$ is a bounded operator on $L^{2}$ for the strong topology, it is bounded also for the weak topology (because of the Riesz representation theorem). We deduce, taking $u_{n_{k}}$ as a test function in (3.1) that

$$
\int_{X}\left|D u_{n_{k}}\right|^{2} d \mu+\int_{X}\left|u_{n_{k}}\right|^{2} d \mu=L\left(u_{n_{k}}, D u_{n_{k}}\right) \rightarrow L(u, D u)=\int_{X}|D u|^{2} d \mu+\int_{X}|u|^{2} d \mu,
$$

which implies the strong convergence of $u_{n_{k}}$ to $u$ and $D u_{n_{k}}$ to $D u$. Consequently, from the uniqueness of solution $u$ for equation $(*)$ we deduce that $u$ is the unique weak limit of $\left\{u_{n}\right\}$ and so the whole sequence $u_{n}$ converges strongly to $u$.

On the other hand, let us prove that $\gamma$-convergence implies Mosco-convergence. We begin by checking condition (M1). For a given $u \in N^{1,2}(\Omega)$ we define the bounded linear form $L$ on $L^{2}(X) \times L^{2}\left(X, \mathbf{R}^{N}\right)$ by

$$
L(v, w):=\int_{\Omega} u v d \mu+\int_{\Omega} D u \cdot w d \mu .
$$

It is clear that, with this choice of linear form $L, u$ is the solution of $(*)$ in $\Omega$. Now if $u_{n}$ is the solution of $(*)$ in $\Omega_{n}$, by $\gamma$-convergence we know that $\hat{u}_{n}$ strongly converges to $\hat{u}$ which proves (M1).

Now we prove (M2). Let $n_{k}$ be a sequence of indices converging to $\infty$ and let $\hat{u}_{k}$ be a sequence in $\mathscr{B}_{\Omega_{n_{k}}}$ that weakly converges in $L^{2}(X) \times L^{2}\left(X, \mathbf{R}^{N}\right)$ to $(u, v)$. We have to prove that $(u, v)=\hat{w}$ for a certain $w \in N^{1,2}(\Omega)$. First, we consider the linear form

$$
L(\varphi, \psi):=\int_{\Omega} u \varphi+v \cdot \psi d \mu
$$

and we denote $w_{k}$ the solution of $(*)$ in $\Omega_{n_{k}}$ associated to $L$. By hypothesis, $\Omega_{n_{k}} \stackrel{\gamma}{\rightarrow} \Omega$ so $\hat{w}_{k}$ strongly converges in $L^{2}(X) \times L^{2}\left(X \times \mathbf{R}^{N}\right)$ to $\hat{w}$, where $w$ is the solution of 
$(*)$ in $\Omega$. Thus, all we have to prove is that $(u, v)=\hat{w}$. Indeed, by $(*)$ we get

$$
\int_{\Omega_{k}} D w_{k} \cdot\left(D w_{k}-D u_{k}\right) d \mu+\int_{\Omega_{k}} w_{k}\left(w_{k}-u_{k}\right) d \mu=L\left(w_{k}-u_{k}, D w_{k}-D u_{k}\right)
$$

and taking the limit strongly in $w_{k}$, weakly in $w_{k}-u_{k}$ (i.e. applying Lemma 8), we obtain (extending $D u_{k}$ by 0 out of $\Omega_{n_{k}}$ ) that

$$
\int_{\Omega} D w \cdot(D w-v) d \mu+\int_{\Omega} w(w-u) d \mu=L(w-u, D w-v),
$$

which implies $u=w$ and $D w=v$, by definition of $L$ (see (3.2)).

\section{Stability for extension domains}

In order to study some stability with respect to a variation of the domain, the family of domains of $X$ has to be endowed with a suitable topology. In this section, following Chenais [8], we choose the topology of characteristic functions.

Definition 14. Let $\Omega_{n}$ be a sequence of domains in $X$. We say that $\Omega_{n}$ converges to the domain $\Omega$ in the characteristic functions topology if the sequence of characteristic functions $\chi_{\Omega_{n}}$ strongly converges in $L^{2}(X)$ to the characteristic function $\chi_{\Omega}$.

We now give the definition of $N^{1,2}$-extension domain.

Definition 15. Let $\Omega \subseteq X$. We say that $\Omega$ is an $N^{1,2}$-extension domain if there is a constant $C>0$ and a bounded linear operator

$$
E: N^{1,2}(\Omega) \rightarrow N^{1,2}(X)
$$

such that $E(u)=u$ in $\Omega$ and

$$
\|E(u)\|_{N^{1,2}(X)} \leq C\|u\|_{N^{1,2}(\Omega)} .
$$

In Section 4.1 we will give more information about extension domains, including sufficient geometric conditions on the boundary of a domain that implies the extension property. Before that, let us prove the following stability result.

Theorem 16. Let $(X, d, \mu)$ be a metric measure space such that the embed$\operatorname{ding} N^{1,2}(X) \hookrightarrow L^{2}(X)$ is compact. Let $\Omega \subseteq X$ and $\Omega_{n} \subseteq X(n \in \mathbf{N})$ be some $N^{1,2}$-extension domains (with same constant $C$ ) such that $\Omega_{n}$ converges to $\Omega$ in the characteristic functions topology. Then $\Omega_{n} \gamma$-converges to $\Omega$.

Proof. This result is contained in [8] for the Euclidean case (see also Theorem 3.7.3 in [18]). There is no deep changes in metric spaces but let us write here the entire proof for sake of completeness.

For all $n$ we denote $\tilde{u}_{n}:=E_{n}\left(u_{n}\right) \in N^{1,2}(X)$ where $E_{n}$ is the extension operator in $\Omega_{n}$ and $u_{n}$ is the solution of $(*)$ in $\Omega_{n}$. By (2.2) we know that

$$
\left\|\tilde{u}_{n}\right\|_{N^{1,2}(X)} \leq C\left\|u_{n}\right\|_{N^{1,2}\left(\Omega_{n}\right)} \leq C\|L\|
$$

thus we can extract a subsequence (not relabeled) that weakly converges in $N^{1,2}(X)$ to a function $u \in N^{1,2}(X)$. By the compact embedding of $N^{1,2}(X)$ into $L^{2}(X)$ we may also assume that $\tilde{u}_{n}$ strongly converges to $u$ in $L^{2}(X)$. We claim that $\chi_{\Omega} u$ is the solution of $(*)$ in $\Omega$. Indeed, for every $\phi \in N^{1,2}(X)$ we have that $\chi_{\Omega_{n}} \phi \in N^{1,2}\left(\Omega_{n}\right)$ 
and since $u_{n}$ is a solution of $(*)$ in $\Omega_{n}$ we can write

$$
\int_{\Omega_{n}} D u_{n} \cdot D \phi d \mu+\int_{\Omega_{n}} u_{n} \phi d \mu=L\left(\chi_{\Omega_{n}} \phi, \chi_{\Omega_{n}} D \phi\right)
$$

which implies

$$
\int_{X} \chi_{\Omega_{n}} D \tilde{u}_{n} \cdot D \phi d \mu+\int_{X} \chi_{\Omega_{n}} \tilde{u}_{n} \phi d \mu=L\left(\chi_{\Omega_{n}} \phi, \chi_{\Omega_{n}} D \phi\right) .
$$

Now up to a subsequence, $\chi_{\Omega_{n}}$ converges to $\chi_{\Omega} \mu$ a.e. in $X$ and is uniformly bounded, so $\chi_{\Omega_{n}} \phi \rightarrow \chi_{\Omega} \phi$ and $\chi_{\Omega_{n}} D \phi \rightarrow \chi_{\Omega} D \phi$ in $L^{2}(X)$. Next, applying Lemma 8 and passing to the limit in (4.1) we obtain

$$
\int_{\Omega} D u \cdot D \phi d \mu+\int_{\Omega} u \phi d \mu=L\left(\chi_{\Omega} \phi, \chi_{\Omega} D \phi\right),
$$

for all $\phi \in N^{1,2}(X)$. In fact, it is true also for all $\phi \in N^{1,2}(\Omega)$ because $\Omega$ is an extension domain. This proves that the restriction of $u$ to $\Omega$ satisfies $(*)$. It remains to prove that $\chi_{\Omega_{n}} D u_{n}$ strongly converges in $L^{2}(X)$ to $\chi_{\Omega} D u$ and this is a consequence of the fact that, since $L$ is continuous for the weak topology of $N^{1,2}(X)$,

$$
\begin{aligned}
\lim _{n \rightarrow+\infty} \int_{\Omega_{n}}\left|D u_{n}\right|^{2} d \mu+\int_{\Omega_{n}}\left|u_{n}\right|^{2} d \mu & =\lim _{n \rightarrow+\infty} L\left(\chi_{\Omega_{n}} u_{n}, \chi_{\Omega_{n}} D u\right) \\
& =L\left(\chi_{\Omega} u, \chi_{\Omega} D u\right)=\int_{\Omega}|D u|^{2} d \mu+\int_{\Omega}|u|^{2} d \mu,
\end{aligned}
$$

so the proof is now complete.

4.1. $N^{1,2}$ - extension domains. It is well known that uniform domains are extension domains for the Sobolev spaces $W^{1, p}(\Omega)$ in Euclidean spaces. This fact was first proved by Jones in [19] for a wide class of domains called $(\varepsilon, \delta)$-domains, and domains which satisfy an $\varepsilon$-cone condition are a particular case. In [2] we can find some geometric conditions for a domain $\Omega$ to be an extension domain for $N^{1,2}$.

Definition 17. A domain $\Omega \subset X$ is A-uniform, $A \geq 1$, if for every pair of points $x, y \in \Omega$ there is a curve $\gamma$ in $\Omega$ connecting $x$ and $y$ such that $\ell(\gamma) \leq A d(x, y)$ and for all $z \in \gamma$,

$$
\operatorname{dist}(z, X \backslash \Omega) \geq \frac{1}{A} \min \left\{\ell\left(\gamma_{x, z}\right), \ell\left(\gamma_{y, z}\right)\right\} .
$$

Here, $\gamma_{x, z}$ and $\gamma_{y, z}$ denotes a subcurve connecting $x$ to $z$ and $y$ to $z$ respectively.

In $[2,5.9]$ it is proved that if $\mu$ supports a weak 2-Poincare inequality on $X$, then every uniform domain is an $N^{1,2}$ - extension domain.

Definition 18. We say that $\Omega$ satisfies a corkscrew condition if there exists $\varepsilon>0$ such that for all $x \in \bar{\Omega}$ and $0<r \leq \operatorname{diam}(\Omega)$, the set $B(x, r) \cap \Omega$ contains a ball of radius $\varepsilon r$.

It is well known that uniform domains satisfy a corkscrew condition (see [2, 4.2]). There are many geometric conditions equivalent to the corkscrew condition. In the following, we will mention the more remarkable ones:

Definition 19. Let $\beta>0$. We say that $\Omega$ satisfies a local $\beta$-shell condition if for every $x \in \bar{\Omega}$ and $0<t \leq r \leq \operatorname{diam}(\Omega)$ there exists a positive constant $C$ such that the shell

$$
S_{t}=\{y \in B(x, r) \cap \Omega: d(y, \partial \Omega) \leq t\}
$$


satisfies

$$
\mu\left(S_{t}\right) \leq C\left(\frac{t}{r}\right)^{\beta} \mu(B(x, r) \cap \Omega) .
$$

Definition 20. Let $0<\varepsilon<1$ and $0<\delta \leq 1$. We say that $\Omega$ satisfies a $(\varepsilon, \delta)$-measure density condition if for every $x \in \bar{\Omega}$ and for each $0<r \leq \operatorname{diam}(\Omega)$,

$$
\mu(\{y \in B(x, r) \cap \Omega: d(y, \partial \Omega)>\varepsilon r\}) \geq \delta \mu(B(x, r) \cap \Omega) .
$$

Theorem 21. [2, 2.8] Let $\mu$ be a doubling measure on $\Omega$ or in $X$. Then the following are equivalent:

(1) $\Omega$ satisfies a corkscrew condition.

(2) $\Omega$ satisfies a local $\beta$-shell condition for some $\beta>0$.

(3) $\Omega$ satisfies a $(\varepsilon, \delta)$-measure density condition for some $\varepsilon>0$ and $\delta>0$.

Now, we can state an extension of a result from Jones [19] to the setting of metric spaces equipped with a doubling measure and supporting a weak 2-Poincaré inequality.

Theorem 22. $[2,5.6]$ Let $\mu$ be a doubling measure and $X$ supporting a weak 2Poincaré inequality. Suppose that $\Omega \subset X$ is a domain such that $\mu(\partial \Omega)=0, \bar{\Omega}$ satisfies one of the conditions in Theorem 21 and that for some $\alpha>0$, the measure given by the density $d \nu(y):=(d(y, \partial \Omega))^{\alpha} d \mu(y)$ supports a weak 2-Poincaré inequality on $\bar{\Omega}$. Then, $\Omega$ is an $N^{1,2}$-extension domain, and moreover the following local estimates for the extension operator hold: for bounded $\Omega$ there exist $\lambda>0$ and $C>0$ such that for all balls $B$ with radius at most $\operatorname{diam}(\Omega)$ and a center in $\bar{\Omega}$

$$
\|E(u)\|_{L^{2}(B)} \leq C\|u\|_{L^{2}(\Omega \cap \lambda B)} \text { and }\|D E(u)\|_{L^{2}(B)} \leq C\|D u\|_{L^{2}(\Omega \cap \lambda B)} .
$$

If $\Omega$ is unbounded, (4.2) holds for balls with radius at most $R$, and $C$ depends on $R$.

A straightforward consequence of Theorem 22 together with Theorem 16 is the following.

Theorem 23. Let $(X, d, \mu)$ be a metric measure space, and let $\Omega_{n} \subseteq X$ and $\Omega \subseteq$ $X$ be some domains satisfying the conditions of Theorem 22 with uniform constants. Suppose also that the sequence $\Omega_{n}$ converges to a domain $\Omega$ in the characteristic function topology. Then $\Omega_{n} \gamma$-converges to $\Omega$.

Remark 24. For instance the class of $\delta$-Reifenberg-flat domains in $\mathbf{R}^{N}$, that are considered in [24] and also in [23] in a more general setting, satisfies any of the conditions in Theorem 21.

\section{Stability under quasiconformal deformations}

In [4] it is proved (in particular) that any sequence of connected and simply connected domains in $\mathbf{R}^{2}$ that converges for the complementary Hausdorff distance is stable for the Neumann problem. This suggests that the regularity of the boundary of the domains is not necessary to have $\gamma$-convergence. In other words the stability might be true for a larger class of domains than the one of extension domains.

In this last section we will prove that the Neumann problem is stable for a sequence of certain classes of quasiballs defined in a general metric space. A quasiball is defined as being the image of the unit ball by a quasiconformal mapping, so this result could be understood as an extension in higher dimensions of the aforementioned result about simply connected domains in $\mathbf{R}^{2}$ (image of the unit disk by a 
conformal mapping). In $\mathbf{R}^{2}$ one can prove that if $\Omega_{k}$ is a sequence of simply connected domains that converges for the complementary Hausdorff distance, then the sequence of conformal representations $g_{k}$ of $\Omega_{k}$ converges itself to a conformal mapping $g$ which represents the limit domain (up to fix the image of one point and the sign of the derivative, see [4,3.1]). In the case of quasiconformal mappings we have less rigidity and one cannot expect such a convergence in general. However, we will see in this section that if we assume in addition that the mappings converge in $L^{1}$ to the identity map, then the stability still holds (see Theorem 33).

Quasiconformal mappings are commonly understood to be homeomorphisms that transform infinitesimal balls into infinitesimall ellipsoids of bounded eccentricity. This requirement makes sense in any metric space. Let $f: X \rightarrow Y$ be a homeomorphism between metric spaces $\left(X, d_{X}\right)$ and $\left(Y, d_{Y}\right)$ and define for each $r>0$ and $x \in X$

$$
\begin{aligned}
L_{f}(x, r) & :=\sup \left\{d_{Y}(f(x), f(y)) ; d_{X}(x, y) \leq r\right\}, \\
l_{f}(x, r) & :=\inf \left\{d_{Y}(f(x), f(y)) ; d_{X}(x, y) \geq r\right\}
\end{aligned}
$$

and

$$
H_{f}(x, r):=\frac{L_{f}(x, r)}{l_{f}(x, r)} .
$$

Observe that the ratio $H_{f}(x, r)$ measures the eccentricity of the image of the ball $B(x, r)$ under $f$, and we always have $H_{f}(x, r) \geq 1$.

Definition 25. We say that the homeomorphism $f: X \rightarrow Y$ is

$$
\begin{aligned}
& K \text {-quasiconformal if } \limsup _{r \rightarrow 0} H_{f}(x, r) \leq K \forall x \in X ; \\
& K \text {-quasisymmetric if } H_{f}(x, r) \leq K \forall x \in X, \forall B(x, r) \subseteq X .
\end{aligned}
$$

Actually, this definition of quasisymmetric mapping is not exactly the one that could be commonly found in the literature, but if the space $X$ is doubling and pathwise connected, then our definition is equivalent to the classical one due to a result of Väisälä (see Lemma 4.6 in [16] or [29, 2.9]).

Observe that if $f$ is $K$-quasisymmetric then $f^{-1}$ is also $K$-quasisymmetric. It is also clear from the definitions that every $\mathrm{K}$-quasisymmetric mapping is a $\mathrm{K}$ quasiconformal mapping. It turns out that for a large class of metric spaces the converse is also true.

It was proven by Heinonen and Koskela [15] (see also [21]) that under the following conditions:

(i) $X$ and $Y$ are $Q$-regular spaces with $Q>1$,

(ii) $X$ is proper,

(iii) $Y$ is locally linearly connected,

(iv) $f: X \rightarrow Y$ is a quasiconformal mapping that maps bounded sets to bounded sets.

If $X$ supports a $Q$-Poincaré inequality then $f$ is quasisymmetric. If in addition, $X$ supports a $p$-Poincaré inequality for some $1 \leq p<Q$, then $f$ is not only quasisymmetric but also absolutely continuous, and the pullback measure is $A_{\infty}$-related to $\mu$. Notice that if $X$ is a proper $Q$-regular space with $Q>1$ which supports a $Q$-Poincaré inequality then it is also quasiconvex (see for example Proposition 4.4 in [14]). 
Recall that a measure $\sigma$ is said to be $A_{\infty}$-related to $\mu$ if for each $\varepsilon>0$ there exists $\delta>0$ such that

$$
\mu(E)<\delta \mu(B) \text { implies } \sigma(E)<\varepsilon \sigma(B),
$$

whenever $E$ is a measurable subset of a ball $B$.

Standard assumptions. In the sequel, we will say that $(X, d, \mu, f)$ satisfies the standard assumptions with constants $(Q, K, p)$ when $X$ is a $Q$-regular and complete metric measure space (thus is separable and locally compact) that admits a $p$-Poincaré inequality for some $p<Q$ and $f: \Omega \rightarrow X$ is a $K$-quasisymmetric map between two bounded domains $\Omega \subset X$ and $f(\Omega) \subset X$. We also assume that the pullback measure $\mu_{f}$ of $\mu$ by $f$ is $A_{\infty}$-related to $\mu$, and that $J_{f}$ (the Radon-Nikodym derivative of $\mu_{f}$ with respect to $\mu$ ) satisfies a reverse Hölder condition. This means that for every ball $B \subset X$

$$
\left(f_{B} J_{f}^{1+\varepsilon}\right) \leq C\left(f_{B} J_{f}\right)^{1+\varepsilon}
$$

with $\varepsilon$ defined by $p=1+\varepsilon^{-1}$.

Example 26. $(X, d, \mu, f)$ satisfies the standard assumptions when (i)-(iv) hold and $X$ supports a $p$-Poincaré inequality for some $p<Q$. By Theorem 1.0.1 in [20], if $X$ is in addition complete, we automatically have a weak $p$-Poincaré inequality, for some $1 \leq p<Q$.

Remark 27. It follows from the Hölder inequality that if a space admits a $p$ Poincaré inequality, it admits a $q$-Poincaré inequality for each $q \geq p$. Thus, even if we can choose $\varepsilon$ as small as we want in (5.1), the estimate which gives more information is the one with $p=1+\varepsilon^{-1}$.

Remark 28. It follows from the quasisymmetry of $f$ and $Q$-regularity of $X$ that (5.1) still holds when the ball $B$ is replaced by the pre-image of a ball by $f$.

In [17] (Theorem 9.10) it is proved that quasiconformal mappings between metric spaces of locally $Q$-bounded geometry (see Definition 9.1 in [17]) preserve the Newtonian-Sobolev space $N^{1, Q}$. More precisely, if $f: X \rightarrow Y$ is a quasiconformal homeomorphism between metric spaces of locally $Q$-bounded geometry then

$$
u \in N_{\mathrm{loc}}^{1, Q}(X) \Rightarrow u \circ f^{-1} \in N_{\mathrm{loc}}^{1, Q}(Y) .
$$

Let us notice that if (i)-(iii) hold and $X$ supports a $Q$-Poincaré inequality, then $X$ is of locally $Q$-bounded geometry (see Section 5 in [16]).

In order to prove the main stability result of this section, we will need some technical lemmas. The first Lemma controls the difference $\left|u_{B}-\left(u \circ f^{-1}\right)_{B}\right|$ provided that the set of points that are mapped far away by $f^{-1}$ has small measure compared to the radius of $B$. Recall that $\lambda$ denotes the constant from the Poincaré inequality (see (1.1)).

Lemma 29. Assume that $(X, d, \mu, f)$ satisfies the standard assumptions with constants $(Q, K, p)$ and that $f: \Omega_{1} \rightarrow \Omega_{2}:=f\left(\Omega_{1}\right)$, where $\Omega_{1}$ and $\Omega_{2}$ are two bounded domains of $X$ such that $\Omega_{1} \cap \Omega_{2} \neq \emptyset$. Assume in addition that

$$
\mu\left(\left\{y \in \Omega_{2}: d\left(y, f^{-1}(y)\right) \geq \frac{1}{100} r\right\}\right) \leq \delta r^{Q}
$$


for a positive constant $\delta \leq\left(2 C_{0}\right)^{-1} 100^{-Q}$, and a given radius $r>0$. Then for all $u \in N^{1, p}\left(\Omega_{1}\right)$ and for all balls $B:=B(x, r) \subseteq \Omega_{1} \cap \Omega_{2}$ such that $B(x, \lambda 4 K r) \subseteq \Omega_{1}$ one has that

$$
f^{-1}(B) \subseteq B(x, 4 K r)
$$

and

$$
\left|u_{B}-\left(u \circ f^{-1}\right)_{B}\right| \leq C r\left(f_{B(x, \lambda 4 K r)} \rho_{u}^{p}\right)^{\frac{1}{p}}
$$

where $C$ depends on $p, K$ and the doubling constant of $\mu$.

Proof. Let $B=B(x, r)$ as in the statement of the lemma and let us denote $E:=f^{-1}(B)$. We claim that

$$
B\left(z, \frac{1}{4 K} r\right) \subseteq E \subseteq B(x, 4 K r)
$$

for some point $z \in X$. Let us begin with the first inclusion. We consider the set $\Sigma:=\left\{y \in \Omega_{2} ; d\left(y, f^{-1}(y)\right) \geq \frac{1}{100} r\right\}$ and we take a point $z_{1} \in B(x, \tau r) \backslash \Sigma$ with $\tau:=\left(2 \delta C_{0}\right)^{\frac{1}{Q}}$. Such a point always exists because $\mu(B(x, \tau r)) \geq C_{0}^{-1} \tau^{Q} r^{Q}=2 \delta r^{Q}>$ $\mu(\Sigma)$. In particular, $d\left(z_{1}, f^{-1}\left(z_{1}\right)\right) \leq \frac{r}{100}$ thus $f^{-1}\left(z_{1}\right) \in B\left(x,\left(\tau+\frac{1}{100}\right) r\right) \subseteq B\left(x, \frac{2}{100} r\right)$ by definition of $\tau$ and because by assumption $\delta \leq\left(2 C_{0}\right)^{-1} 100^{-Q}$. Now take a point $y \in B$ such that $d(x, y)=\frac{1}{2} r$. By a similar argument one can choose a point $z_{2}$ in $B(y, \tau r) \backslash \Sigma$ such that $f^{-1}\left(z_{2}\right) \in B\left(y, \frac{2}{100} r\right)$. In particular we have that

$$
d\left(f^{-1}\left(z_{1}\right), f^{-1}\left(z_{2}\right)\right) \geq \frac{r}{2}-\frac{4}{100} r \geq \frac{r}{4}
$$

and

$$
d\left(z_{1}, z_{2}\right) \leq \frac{r}{2}+\frac{1}{50} r \leq \frac{3}{2} r
$$

which implies that $L_{f^{-1}}\left(z_{1}, \frac{3}{2} r\right) \geq \frac{r}{4}$. Since

$$
L_{f^{-1}}\left(z_{1}, \frac{3}{2} r\right) / l_{f^{-1}}\left(z_{1}, \frac{3}{2} r\right)=H\left(x, \frac{3}{2} r\right) \leq K,
$$

we deduce that

$$
l_{f^{-1}}\left(z_{1}, \frac{3}{2} r\right) \geq \frac{r}{4 K}
$$

This proves that $f^{-1}\left(B\left(z_{1}, \frac{3}{2} r\right)\right)$ contains a ball of radius at least $\frac{r}{4 K}$ and so, there exists a $z$ such that

$$
B\left(z, \frac{1}{4 K} r\right) \subseteq f^{-1}\left(B\left(z_{1}, \frac{3}{4} r\right)\right) \subseteq f^{-1}(B) .
$$

Now let us prove the second inclusion in (5.5). We keep the same point $z_{1} \in$ $B(x, \tau r) \backslash K$ and following the previous arguments, we find a point $z_{2}$ lying in $B(y, \tau r) \backslash$ $\Sigma$ where this time $y$ is chosen satisfying $d(x, y)=2 r$. By construction we get

$$
d\left(f^{-1}\left(z_{1}\right), f^{-1}\left(z_{2}\right)\right) \leq 2 r+\frac{22}{100} r=\frac{222}{100} r
$$

and

$$
d\left(z_{1}, z_{2}\right) \geq 2 r-\frac{22}{100} r=\frac{178}{100} r
$$


These two estimates implies $l_{f^{-1}}\left(z_{1}, \frac{178}{100} r\right) \leq \frac{222}{100} r$. Therefore $L_{f^{-1}}\left(z_{1}, \frac{178}{100} r\right) \leq K \frac{222}{100} r$ and so we deduce that there exists a point $z^{\prime}$ such that

$$
f^{-1}(B) \subseteq f^{-1}\left(B\left(\left(z_{1}, \frac{178}{100} r\right)\right) \subseteq B\left(z^{\prime}, \frac{222}{100} K r\right) \subseteq B(x, 4 K r)\right.
$$

and claim (5.5) is proved.

Now let us denote $B_{0}:=B(x, 4 K r)$. Observe that if $g$ is an upper gradient for $u$, then for any constant $a>0, g$ is still an upper gradient for $u+a$. In addition $(u+a) \circ f^{-1}=u \circ f^{-1}+a$. Therefore, to prove (5.4) we can assume without loss of generality that

$$
f_{B_{0}} u d \mu=0 \text {. }
$$

By Theorem 4, we get in particular that

$$
\left(f_{B_{0}}|u|^{p} d \mu\right)^{\frac{1}{p}} \leq C r\left(f_{\lambda B_{0}} g^{p} d \mu\right)^{\frac{1}{p}} .
$$

Our aim now is to estimate

$$
\left|u_{B}-\left(u \circ f^{-1}\right)_{B}\right| \leq\left|u_{B}\right|+\left|\left(u \circ f^{-1}\right)_{B}\right| .
$$

Using $p$-Poincaré inequality and minding that $u_{B_{0}}=0$ we get

$$
\left|u_{B}\right|=\left|u_{B}-u_{B_{0}}\right| \leq \frac{\mu\left(B_{0}\right)}{\mu(B)} f_{B_{0}}\left|u-u_{B_{0}}\right| d \mu \leq C r\left(f_{\lambda B_{0}} g^{p} d \mu\right)^{\frac{1}{p}} .
$$

So we are left to estimate $\left|\left(u \circ f^{-1}\right)_{B}\right|$. Let $\mu_{f}$ be the pull-back measure of $\mu$ by $f$, that is,

$$
\mu_{f}(E)=\mu\left(f^{-1}(E)\right),
$$

for any Borel set $E$ of $X$. Recall that under our assumptions we know that $\mu_{f}$ is absolutely continuous with respect to $\mu$ and we have

$$
\mu_{f}=J_{f} \mu .
$$

Recall also that by definition of $\varepsilon$ we have $p^{\prime}=\varepsilon+1$ where $p^{\prime}$ is the conjugate of $p$. From the reverse Hölder's inequality satisfied by $J_{f}$ it follows

$$
\begin{aligned}
\left|\left(u \circ f^{-1}\right)_{B}\right| & \leq \frac{1}{\mu(B)} \int_{E}|u| J_{f} d \mu \leq\left(\frac{1}{\mu(B)} \int_{E}|u|^{p}\right)^{\frac{1}{p}}\left(\frac{1}{\mu(B)} \int_{E} J_{f}^{\varepsilon+1} d \mu\right)^{\frac{1}{\varepsilon+1}} \\
& \leq\left(\frac{\mu\left(B_{0}\right)}{\mu(B)}\right)^{\frac{1}{p}}\left(f_{B_{0}}|u|^{p}\right)^{\frac{1}{p}}\left(\frac{\mu(E)}{\mu(B)}\right)^{\frac{1}{\varepsilon+1}}\left(f_{E} J_{f}^{\varepsilon+1} d \mu\right)^{\frac{1}{\varepsilon+1}} \\
& \leq C(\varepsilon, K, \mu) r\left(f_{\lambda B_{0}} g^{p}\right)^{\frac{1}{p}} f_{E} J_{f} d \mu \leq C r\left(f_{\lambda B_{0}} g^{p}\right)^{\frac{1}{p}} .
\end{aligned}
$$

Let us observe that the control of $f_{E} J_{f} d \mu$ in (5.8) uses (5.5) and the doubling property of $\mu$. Furthermore, to get inequality (5.8) we have used (5.7), (5.1), (5.5) and Remark 28. The proof of the lemma is now complete.

Our stability result (Theorem 33) will rely on a "key lemma" that allows us to compare two functions defined in different domains, and say that they are close in the Newtonian space norm when the domains are close enough for a suitable topology. 
We will need some classical Whitney type coverings and we refer for instance to [1] for a proof of the following Lemma.

Lemma 30. Let $\Omega \subseteq X$ be an open set such that $\partial \Omega$ is not empty, and let us define for $\varepsilon>0$ the sets

$$
V_{\varepsilon}:=\left\{x \in \Omega ; 0<d\left(x, \Omega^{c}\right) \leq \varepsilon\right\} \subseteq \Omega .
$$

Then for any constant $\lambda, K \geq 1$ there exists a countable family of balls $\left\{B_{i}\right\}_{i \in I}$ of center $x_{i}$ and radius $r_{i}$ satisfying

(i) $V_{\varepsilon} \subseteq \cup_{i \in I} B_{i} \subseteq V_{\frac{9}{8} \varepsilon}$,

(ii) $r_{i} 100 \lambda K \leq d\left(x_{i}, \Omega^{c}\right) \leq r_{i} 200 \lambda K$ for all $i \in I$,

(iii) the balls in the family $\left\{\frac{1}{2} B_{i}\right\}_{i \in I}$ are pairwise disjoint,

(iv) if $B_{j} \cap B_{j} \neq \emptyset$, then $r_{i} \leq 4 r_{j}$, and

(v) $\sum_{i \in I} \chi_{10 B_{i}}(x) \leq M$ where $M$ depends only on the doubling constant of $\mu$.

By standard techniques there exists a partition of unity $\left\{\varphi_{i}\right\}_{i \in I}$ associated to $\left\{B_{i}\right\}_{i \in I}$ and satisfying

(i) $\sum_{i \in I} \varphi_{i}(x)=1$ on $V_{\varepsilon}$,

(ii) $\operatorname{supp}\left(\varphi_{i}\right) \subset 2 B_{i}$ for all $i \in I$,

(iii) $0 \leq \varphi_{i} \leq 1$ for all $i \in I$, and

(iv) $\varphi_{i}$ is $C / r_{i}$-Lipschitz for all $i \in I$.

From this partition of unity on $V_{\varepsilon} \subseteq \Omega$ we would like to obtain a partition of unity on all $\Omega$. To this aim, let us define

$$
\psi(x):=l\left(d\left(x, \Omega^{c}\right) / \varepsilon\right)
$$

where $l$ is a $C$-Lipschitz function equal to 0 in $[0,1 / 2]$, equal to 1 in $[1,+\infty)$ and $l^{\prime}(x) \leq C$. Observe that by construction $\psi$ is $C / \varepsilon$-Lipschitz, equal to 0 on $V_{\varepsilon / 2}$ and $\psi+\sum_{i \in I} \varphi_{i}>0$ on $\Omega$. Now, we define

$$
\theta_{i}:=\frac{\varphi_{i}}{\psi+\sum_{i \in I} \varphi_{i}} \text { for } i \in I \quad \text { and } \quad \psi_{0}:=\frac{\psi}{\psi+\sum_{i \in I} \varphi_{i}} \text { for } i \in I .
$$

We now have a partition of unity in $\Omega$, and the following properties are easily checked:

(i) $\psi_{0}+\sum_{i \in I} \theta_{i}=1$ on $\Omega$.

(ii) $\operatorname{supp}\left(\theta_{i}\right) \subset 2 B_{i}$ for all $i \in I$.

(iii) $\operatorname{supp}\left(\psi_{0}\right) \subset \Omega \backslash V_{\varepsilon / 2}$.

(iv) $\psi_{0}$ is $C / \varepsilon$-Lipschitz.

(v) $\psi_{0}=1$ on $\Omega \backslash V_{\varepsilon}$.

We will need also the following result.

Lemma 31. Let $\Omega \subset X$ be an open set. Then, for any $q \geq p$ and for any $u \in N^{1, q}(\Omega)$ one has

$$
\left\|u \psi_{0}+\sum_{i \in I} u_{B_{i}} \theta_{i}\right\|_{N^{1, q}(A)} \leq C\|u\|_{N^{1, q}(W(A))},
$$

where $W(A):=A \cup \bigcup_{i \in I ; 2 B_{i} \cap A \neq \emptyset} 10 \lambda B_{i}$ for any Borel set $A \subset \Omega$.

Proof. The proof relies on the classical Whitney argument. The adaptation in a metric space framework is inspired by the proof of Theorem 5.6 in [2]. Set

$$
f(x):=u(x) \psi_{0}(x)+\sum_{i \in I} u_{B_{i}} \theta_{i}(x),
$$


and let us first control the $L^{q}$-norm of $f$. We denote $I_{A}:=\left\{i \in I: \exists y \in A: \theta_{i}(y) \neq 0\right\}$ and for any $x \in \Omega$ we denote $I_{x}:=\left\{i \in I ; \theta_{i}(x) \neq 0\right\}$. Using the fact that $\sharp I_{x} \leq C$ and applying Fubini's theorem, we obtain the following chain of inequalities:

$$
\begin{aligned}
\left\|\sum_{i \in I} u_{B_{i}} \theta_{i}\right\|_{L^{q}(A)}^{q} & =\int_{A}\left|\sum_{i \in I_{x}} u_{B_{i}} \theta_{i}(x)\right|^{q} d \mu \leq C \int_{A} \sum_{i \in I_{x}}\left|u_{B_{i}} \theta_{i}(x)\right|^{q} d \mu \\
& \leq C \sum_{I_{A}}\left|u_{B_{i}}\right|^{q} \int_{\operatorname{supp}\left(\theta_{i}\right)} d \mu \leq C \sum_{I_{A}}\left|u_{B_{i}}\right|^{q} \mu\left(B_{i}\right) \\
& \leq C \sum_{I_{A}} \int_{B_{i}}|u|^{q} d \mu \leq C \int_{W(A)}|u|^{q} d \mu .
\end{aligned}
$$

Therefore, since $\psi_{0} \leq 1$ and $A \subseteq W(A)$,

$$
\|f\|_{L^{q}(A)} \leq\left\|u \psi_{0}\right\|_{L^{q}(A)}+C\left\|\sum_{i \in I} u_{B_{i}} \theta_{i}\right\|_{L^{q}(W(A))} \leq C\|u\|_{L^{q}(W(A))} .
$$

Now we have to control the gradient of $f$. For this purpose we need first to find a suitable upper gradient for $f$. Let $\gamma$ be a curve connecting two points $x$ and $y$. Up to split $\gamma$ into parts we may assume that $|\gamma| \subseteq 2 B_{i_{0}}$ for some $i_{0} \in I$, or $|\gamma| \subseteq \Omega \backslash \cup_{i \in I} 2 B_{i}$. In the latter case we have that $f=u$ and so, for any upper gradient $g$ of $u$ one has

$$
|f(x)-f(y)| \leq \int_{\gamma} g .
$$

Consequently, it is enough to consider the first case when $|\gamma| \subseteq 2 B_{i_{0}}$ for some $i_{0} \in I$. First, we can add and subtract the constant $u_{10 B_{i_{0}}}$ and use that $\psi_{0}+\sum_{i \in I} \theta_{i}(x)=1$ to write $f(x)-f(y)$ in the following fashion:

$$
\begin{aligned}
f(x)-f(y)= & \underbrace{\left(u(x)-u_{10 B_{i_{0}}}\right)\left(\psi_{0}(x)-\psi_{0}(y)\right)}_{(\star)}+\psi_{0}(y)(u(x)-u(y)) \\
& +\underbrace{\sum_{i \in I}\left(u_{B_{i}}-u_{10 B_{i_{0}}}\right)\left(\theta_{i}(x)-\theta_{i}(y)\right)}_{(\star \star)} .
\end{aligned}
$$

First, let us estimate $(\star \star)$. We have that

$$
\begin{aligned}
\left|\sum_{i \in I}\left(u_{B_{i}}-u_{10 B_{i_{0}}}\right)\left(\theta_{i}(x)-\theta_{i}(y)\right)\right| & \leq \sum_{i \in I}\left|u_{B_{i}}-u_{10 B_{i_{0}}}\right|\left|\theta_{i}(x)-\theta_{i}(y)\right| \\
& \leq C d(x, y) \sum_{i \in I_{x} \cup I_{y}}\left|u_{B_{i}}-u_{10 B_{i_{0}}}\right| \frac{1}{r_{i}} \\
& \leq C d(x, y) \frac{1}{r_{i_{0}}} \sum_{i \in I_{x} \cup I_{y}}\left|u_{B_{i}}-u_{10 B_{i_{0}}}\right| .
\end{aligned}
$$

We use now $q$-Poincaré inequality (which holds since $q \geq p$ ) to obtain that for all $i \in I_{x} \cup I_{y}$, that is, for all $i \in I$ with $2 B_{i} \cap B_{i_{0}} \neq \emptyset$ (and such balls $B_{i}$ are contained in $\left.10 B_{i_{0}}\right)$,

$$
\left|u_{B_{i}}-u_{10 B_{i_{0}}}\right| \leq C r_{i_{0}}\left(f_{10 \lambda B_{i_{0}}} g^{q}\right)^{\frac{1}{q}} .
$$


This last estimate together with the fact that $\sharp\left\{i \in I ; 2 B_{i} \cap B_{i_{0}} \neq \emptyset\right\} \leq C$ yields

$$
\left|\sum_{i \in I}\left(u_{B_{i}}-u_{10 B_{i_{0}}}\right)\left(\theta_{i}(x)-\theta_{i}(y)\right)\right| \leq C d(x, y)\left(f_{10 \lambda B_{i_{0}}} g^{q}\right)^{\frac{1}{q}} .
$$

On the other hand since $\psi_{0}$ is either $C / r_{i_{0}}$-Lipschitz or equal to 0 in $B_{i_{0}}$ we have that

$$
\left|u(x)-u_{10 B_{i_{0}}}\right|\left|\psi_{0}(x)-\psi_{0}(y)\right| \leq C d(x, y) \frac{1}{r_{i_{0}}}\left|u(x)-u_{10 B_{i_{0}}}\right| .
$$

Keeping in mind that $\left|\psi_{0}(y)\right| \leq 1$, all together we have proved

$$
|f(x)-f(y)| \leq|u(x)-u(y)|+C d(x, y)\left[\left(f_{10 \lambda B_{i_{0}}} g^{q}\right)^{\frac{1}{q}}+\frac{1}{r_{i_{0}}}\left|u(x)-u_{10 B_{i_{0}}}\right|\right] \text {. }
$$

From those last estimates, up to a new choice of constant $C$, we claim that the function

$$
G(x):=C g(x)+C \sum_{i \in I ; x \in B_{i}}\left[\left(f_{10 \lambda B_{i}} g^{q}\right)^{\frac{1}{q}}+\frac{1}{r_{i}}\left|u(x)-u_{10 B_{i}}\right|\right]
$$

is an upper gradient for $f$.

Indeed, for any couple of points $x$ and $y$ belonging to $\Omega$, and for any curve $\gamma$ connecting $x$ to $y$ we can split $\gamma$ into a finite number of parts $\gamma_{k}$ in such a way that $\gamma_{k}$ is connected, $\gamma_{k} \subset 2 B_{k}$ for some $k \in I$, and $\gamma_{0} \subset \Omega \backslash \cup_{i \in I} 2 B_{i}$. Let $x_{k}$ and $y_{k}$ be the two endpoints of $\gamma_{k}$, for each $k$. First observe that for any $k$ and for any $z \in \gamma_{k}$ it holds

$$
\left|u\left(x_{k}\right)-u_{10 B_{k}}\right| \leq\left|u(z)-u_{10 B_{k}}\right|+\int_{\gamma_{k}} g
$$

Thus

$$
\begin{aligned}
\sum_{k} \frac{d\left(x_{k}, y_{k}\right)}{r_{k}}\left|u\left(x_{k}\right)-u_{10 B_{k}}\right| & \leq \sum_{k} \frac{d\left(x_{k}, y_{k}\right)}{r_{k}}\left(\left|u(z)-u_{10 B_{k}}\right|+\int_{\gamma_{k}} g\right) \\
& \leq C \int_{\gamma} g+\sum_{k} \frac{d\left(x_{k}, y_{k}\right)}{r_{k}} \inf _{z \in \gamma_{k}}\left|u(z)-u_{10 B_{k}}\right| .
\end{aligned}
$$

We deduce that

$$
\begin{aligned}
& |f(x)-f(y)| \leq\left|f\left(x_{0}\right)-f\left(y_{0}\right)\right|+\sum_{k}\left|f\left(x_{k}\right)-f\left(y_{k}\right)\right| \\
& \stackrel{(5.11)}{\leq} \int_{\gamma_{0}} g+C \sum_{k}\left|u\left(x_{k}\right)-u\left(y_{k}\right)\right|+d\left(x_{k}, y_{k}\right)\left[\left(f_{10 \lambda B_{k}} g^{q}\right)^{\frac{1}{q}}+\frac{1}{r_{k}}\left|u\left(x_{k}\right)-u_{10 B_{k}}\right|\right] \\
& \leq C \int_{\gamma} g+C \sum_{k} \int_{\gamma_{k}} g+C \sum_{k} \ell\left(\gamma_{k}\right)\left[\left(f_{10 \lambda B_{k}} g^{q}\right)^{\frac{1}{q}}+\frac{1}{r_{k}} \inf _{z \in \gamma_{k}}\left|u(z)-u_{10 B_{k}}\right|\right] \\
& \leq C \int_{\gamma} g+C \sum_{k} \int_{\gamma_{k}}\left(\sum_{i \in I ; z \in B_{i}}\left[\left(f_{10 \lambda B_{i}} g^{q}\right)^{\frac{1}{q}}+\frac{1}{r_{i}}\left|u(z)-u_{10 B_{i}}\right|\right]\right) d \mathscr{H}^{1}(z) \\
& \leq \int_{\gamma} G(z) d \mathscr{H}^{1}(z) .
\end{aligned}
$$


In the penultimate inequality we have used that for every $z \in B_{k}$,

$$
\frac{1}{r_{k}}\left|u(z)-u_{10 B_{k}}\right| \leq \sum_{i \in I ; z \in B_{i}} \frac{1}{r_{i}}\left|u(z)-u_{10 B_{i}}\right|
$$

and

$$
\left(f_{10 \lambda B_{k}} g^{q}\right)^{\frac{1}{q}} \leq \sum_{i \in I ; z \in B_{i}}\left(f_{5 \lambda B_{i}} g^{q}\right)^{\frac{1}{q}} .
$$

We have then proved that $G(x)$ defined in (5.12) is an upper gradient for $f$. Now to finish the proof of the lemma, it suffices to control the $L^{q}$-norm of $G$. We have that

$$
\|G\|_{L^{q}(A)} \leq C\|g\|_{L^{q}(A)}+C\left\|\sum_{i \in I_{x}} f_{10 \lambda B_{i}} g^{q}\right\|_{L^{q}(A)}+\left\|\sum_{i \in I ; x \in B_{i}} \frac{1}{r_{i}}\left|u(x)-u_{10 B_{i}}\right|\right\|_{L^{q}(A)} .
$$

On one hand, by an argument similar to (5.9), we get

$$
\begin{aligned}
\left\|C \sum_{i \in I_{x}} f_{10 \lambda B_{i}} g^{q}\right\|_{L^{q}(A)}^{q} & \leq C \int_{A} \sum_{i \in I_{x}}\left(f_{10 \lambda B_{i}} g^{q}\right)^{q} d \mu(x) \\
& \leq C \sum_{I_{A}}\left(f_{10 \lambda B_{i}} g^{q}\right)^{q} \int_{\operatorname{supp}\left(\theta_{i}\right)} d \mu(x) \\
& \leq C \sum_{I_{A}}\left(f_{10 \lambda B_{i}} g^{q}\right)^{q} \mu\left(B_{i}\right) \\
& \leq C \sum_{I_{A}} \int_{10 \lambda B_{i}} g^{q} d \mu \leq C \int_{W(A)} g^{q} d \mu .
\end{aligned}
$$

On the other hand, using Theorem 4 and Fubini's theorem we obtain

$$
\begin{aligned}
\left\|C \sum_{i \in I_{x}} \frac{1}{r_{i}}\left|u(x)-u_{10 B_{i}}\right|\right\|_{L^{q}(A)}^{q} & \leq C \int_{A} \sum_{i \in I_{x}} \frac{1}{r_{i}^{q}}\left|u(x)-u_{10 B_{i}}\right|^{q} d \mu(x) \\
& \leq C \sum_{I_{A}} \frac{1}{r_{i}^{q}} \int_{10 B_{i}}\left|u(x)-u_{10 B_{i}}\right|^{q} d \mu(x) \\
& \leq C \sum_{I_{A}} \int_{10 \lambda B_{i}} g^{q}(x) d \mu(x) \\
& \leq C \int_{W(A)} g^{q}(x) d \mu(x),
\end{aligned}
$$

which finishes the proof of the lemma.

We will denote by $d_{H}$ the Hausdorff distance between two non empty closed sets $A, B$ of $X$ defined by

$$
d_{H}(A, B):=\sup _{x \in A} d(x, B)+\sup _{x \in B} d(x, A),
$$

and $d_{H}^{c}$ will denote the complementary Hausdorff distance between two open sets

$$
d_{H}^{c}\left(\Omega_{1}, \Omega_{2}\right):=d_{H}\left(\Omega_{1}^{c}, \Omega_{2}^{c}\right) .
$$

We are now ready to prove the analogue of the so-called "key lemma" in [23] which leads to the desired $\gamma$-convergence result. 
Lemma 32. For any constants $K, Q$ and $C_{0}$ there exists $\varepsilon_{0}$ and $c$ such that the following holds. Let $\Omega_{1}, \Omega_{2}$ be two bounded domains in $X$. Assume that $(X, d, \mu, f)$ satisfies the standard assumptions with $f: \Omega_{1} \rightarrow \Omega_{2}$. Assume in addition that

$$
\int_{\Omega_{2}} d\left(f^{-1}(x), x\right) d \mu+d_{H}^{c}\left(\Omega_{1}, \Omega_{2}\right) \leq \varepsilon
$$

for some $\varepsilon \in(0,1)$ satisfying $\varepsilon<\min \left(10^{-10} \operatorname{diam}\left(\Omega_{1}\right), \varepsilon_{0}\right)$. Then for every $u \in$ $N^{1, Q}\left(\Omega_{1}\right)$, there exists $\tilde{u} \in N^{1, Q}\left(\Omega_{2}\right)$ such that $u=\tilde{u}$ in $\Omega_{\varepsilon}$ (defined below) and

$$
\|\tilde{u}\|_{N^{1, Q}\left(\Omega_{2}\right)} \leq\|u\|_{N^{1, Q}\left(\Omega_{1}\right)}+C\|u\|_{N^{1, Q}\left(f^{-1}\left(\Lambda_{\varepsilon}\right)\right)},
$$

where

$$
\Lambda_{\varepsilon}:=\left\{x \in \Omega_{2} ; d\left(x, \Omega_{2}^{c}\right) \leq c \varepsilon^{\frac{1}{1+Q}}\right\} \quad \text { and } \quad \Omega_{\varepsilon}:=\left\{x \in \Omega_{2} ; d\left(x, \Omega_{2}^{c}\right) \geq c \varepsilon^{\frac{1}{1+Q}}\right\} .
$$

If in addition $f$ is bi-Lipschitz, then for every $q \geq p$ and for every $u \in N^{1, q}\left(\Omega_{1}\right)$ there exists $\tilde{u} \in N^{1, q}\left(\Omega_{2}\right)$ such that $u=\tilde{u}$ in $\Omega_{\varepsilon}$ and (5.17) holds with $N^{1, q}$ instead of $N^{1, Q}$.

Proof. Let $\varepsilon \in(0,1)$ and let $u \in N^{1, Q}\left(\Omega_{1}\right)$. Observe that $\Omega_{2} \subseteq \Lambda_{\varepsilon} \cup \Omega_{\varepsilon}$ and by (5.16), together with the fact that $\varepsilon<1$, we have $\Omega_{\varepsilon} \subset \Omega_{1} \cap \Omega_{2}$. On the other hand, recall that under our standard assumptions, quasiconformal mappings preserve the Newtonian-Sobolev space $N^{1, Q}$ (see discussion after Remark 28). Therefore, since $f$ is $K$-quasisymmetric (in particular, it is also $K$-quasiconformal), we get that $v:=u \circ f^{-1} \in N^{1, Q}\left(\Omega_{2}\right)$ and for any Borel set $A \subseteq \Omega_{2}$ we have that

$$
\|v\|_{N^{1, Q}(A)} \leq C\|u\|_{N^{1, Q}\left(f^{-1}(A)\right)} .
$$

If in addition $f$ is bi-Lipschitz and $u \in N^{1, q}$, then (5.18) holds with $N^{1, q}$ instead of $N^{1, Q}$. So roughly speaking, our aim is to keep $\tilde{u}=u$ in $\Omega_{\varepsilon}$ and set $\tilde{u}=v$ in $\Lambda_{\varepsilon}$. A Whitney decomposition is the appropriate tool for glueing the two functions.

Indeed, let $\left\{B_{i}\right\}_{i \in I}$ be the Whitney decomposition given by Lemma 30 associated to

$$
\Lambda_{\varepsilon}^{\prime}:=\left\{x \in \Omega_{2} ; d\left(x, \Omega_{2}^{c}\right) \leq r(\varepsilon)\right\},
$$

where $r(\varepsilon)=\frac{c}{10} \varepsilon^{\frac{1}{Q+1}}$ and where $c$ will be defined later depending on $C_{0}, K, \lambda$ and $Q$. Let $\psi_{0}$ and $\left\{\theta_{i}\right\}_{i \in I}$ be the associated partition of unity such that $\psi_{0}+\sum_{i \in I} \theta_{i}=1$ on $\Omega_{2}$ and where the sum is locally finite. Then we define

$$
\tilde{u}:=u \psi_{0}+\sum_{i \in I} v_{B_{i}} \theta_{i}
$$

where, as usual,

$$
v_{B_{i}}:=\frac{1}{\mu\left(B_{i}\right)} \int_{B_{i}} v(x) d x .
$$

By construction of $\psi_{0}$ we have $u=\tilde{u}$ in $\Omega_{\varepsilon}$. It remains to prove (5.17), which will follow from Lemma 31 and Lemma 29.

Let us first control the $L^{Q}$-norm of $\tilde{u}$. Using Lemma 31 and (5.18) we get

$$
\begin{aligned}
\|\tilde{u}\|_{L^{Q}\left(\Omega_{2}\right)} & \leq\left\|u \psi_{0}\right\|_{L^{Q}\left(\Omega_{2}\right)}+\left\|\sum_{i \in I} v_{B_{i}} \theta_{i}\right\|_{L^{Q}\left(\Omega_{2}\right)} \\
& \leq\|u\|_{L^{Q}\left(\Omega_{1}\right)}+C\|v\|_{L^{Q}\left(\Lambda_{\varepsilon}\right)} \leq\|u\|_{L^{Q}\left(\Omega_{1}\right)}+C\|u\|_{L^{Q}\left(f^{-1}\left(\Lambda_{\varepsilon}\right)\right)} .
\end{aligned}
$$

Now we have to control the norm of the gradient of $u$. Let us first observe that if we split $I$ into $I_{1} \cup I_{2}$ with

$$
I_{1}:=\left\{i \in I ; \operatorname{supp}\left(\psi_{0}\right) \cap 2 B_{i} \neq 0\right\}, \quad I_{2}:=I \backslash I_{1},
$$


and if we set

$$
A_{1}:=\bigcup_{i \in I_{1}} 2 B_{i} \quad \text { and } \quad A_{2}:=\bigcup_{i \in I_{2}} 2 B_{i}
$$

then

$$
\|\tilde{u}\|_{N^{1, Q}\left(\Omega_{2}\right)} \leq\|\tilde{u}\|_{N^{1, Q}\left(\Omega_{\varepsilon}\right)}+\|\tilde{u}\|_{N^{1, Q}\left(A_{1}\right)}+\|\tilde{u}\|_{N^{1, Q}\left(A_{2}\right)} .
$$

On the other hand, observe that $\tilde{u}=u$ on $\Omega_{\varepsilon}$, and $\tilde{u}(x)=\sum_{i \in I} v_{B_{i}} \theta_{i}(x)$ on $A_{2}$. Therefore, if we apply Lemma 31 to $v$ and then (5.18) we obtain

$$
\|\tilde{u}\|_{N^{1, Q}\left(\Omega_{2}\right)} \leq\|u\|_{N^{1, Q}\left(\Omega_{\varepsilon}\right)}+\|\tilde{u}\|_{N^{1, Q}\left(A_{1}\right)}+C\|u\|_{N^{1, Q}\left(f^{-1}\left(A_{2}\right)\right)} .
$$

Thus, since $f^{-1}\left(A_{2}\right) \subset f^{-1}\left(\Lambda_{\varepsilon}\right)$, all we have to prove is that

$$
\|\tilde{u}\|_{N^{1, Q}\left(A_{1}\right)} \leq\|u\|_{N^{1, Q}\left(f^{-1}\left(\Lambda_{\varepsilon}\right)\right)} .
$$

For this purpose, we have to find a suitable upper gradient for $\tilde{u}$ in $A_{1}$ and control its norm. Let $\gamma$ be a curve connecting two points $x$ and $y$ of $A_{1}$. We argue as in the proof of Lemma 31. Up to split $\gamma$ into parts we may assume that $|\gamma| \subseteq B_{i_{0}}$ for some $i_{0} \in I_{1}$. In the latter situation we can add and subtract the constant $v_{10 B_{i_{0}}}$ and use that $\psi_{0}+\sum_{i \in I} \theta_{i}(x)=1$ to write (after some computations)

$$
\begin{aligned}
f(x)-f(y)= & \underbrace{\left(u(x)-v_{10 B_{i_{0}}}\right)\left(\psi_{0}(x)-\psi_{0}(y)\right)}_{(\diamond)}+\psi_{0}(y)(u(x)-u(y)) \\
& +\underbrace{\sum_{i \in I}\left(v_{B_{i}}-v_{10 B_{i_{0}}}\right)\left(\theta_{i}(x)-\theta_{i}(y)\right)}_{(\diamond \diamond)} .
\end{aligned}
$$

First, let us control the term $(\diamond)$, which stand actually for the key point of the proof. Recall first that there exists a constant $a \geq 1$ depending on $K, \lambda$ and $Q$ such that the radius of $10 B_{i_{0}}$ denoted $r_{i_{0}}$ satisfies

$$
a^{-1} r(\varepsilon) \leq r_{i_{0}} \leq \operatorname{ar}(\varepsilon) .
$$

Now, using (5.16) and Tchebychev's inequality we obtain that

$$
\begin{aligned}
\mu\left(\left\{z \in \Omega_{2} ; d\left(z, f^{-1}(z)\right) \geq \frac{1}{100} r_{i_{0}}\right\}\right) & \leq \mu\left(\left\{z \in \Omega_{2} ; d\left(z, f^{-1}(z)\right) \geq \frac{1}{100 a} r(\varepsilon)\right\}\right) \\
& \leq \frac{100 a}{r(\varepsilon)} \int_{\Omega_{2}} d\left(z, f^{-1}(z)\right) d \mu \leq \frac{100 a}{r(\varepsilon)} \varepsilon .
\end{aligned}
$$

Now if $\delta:=\left(2 C_{0}\right)^{-1} 100^{-Q}$ (the constant of Lemma 29), we can define $r(\varepsilon)$ in such a way that

$$
\frac{100 a}{r(\varepsilon)} \varepsilon=\delta\left(\frac{r(\varepsilon)}{a}\right)^{Q} \Rightarrow r(\varepsilon)=\left(\frac{100 a^{Q+1}}{\delta}\right)^{\frac{1}{Q+1}} \varepsilon^{\frac{1}{Q+1}},
$$

(which gives at the same time the definition of constant $c$ ) and now (5.21) becomes

$$
\mu\left(\left\{z \in \Omega_{2} ; d\left(z, f^{-1}(z)\right) \geq \frac{1}{100} r_{i_{0}}\right\}\right) \leq \delta\left(\frac{r(\varepsilon)}{a}\right)^{Q} \leq \delta r_{i_{0}}^{Q} .
$$


Subsequently, we can apply Lemma 29 with $B:=10 B_{i_{0}}$ to prove the following estimate

$$
\begin{aligned}
\left|u(x)-v_{10 B_{i_{0}}}\right| & \leq\left|u(x)-u_{10 B_{i_{0}}}\right|+\left|u_{10 B_{i_{0}}}-v_{10 B_{i_{0}}}\right| \\
& \leq\left|u(x)-u_{10 B_{i_{0}}}\right|+C r_{i_{0}}\left(f_{50 K \lambda B_{i_{0}}} g^{Q} d z\right)^{\frac{1}{Q}} .
\end{aligned}
$$

Since $\psi_{0}$ is $C / r_{i_{0}}$-Lipschitz and $d(x, y) \leq 2 r_{i_{0}}$,

$$
\left|u(x)-v_{10 B_{i 0}}\right|\left|\psi_{0}(x)-\psi_{0}(y)\right| \leq C\left|u(x)-u_{10 B_{i_{0}}}\right|+C r_{i_{0}}\left(f_{50 K \lambda B_{i_{0}}} g^{Q} d z\right)^{\frac{1}{Q}}
$$

Let us control now the term $(\diamond \diamond)$. First, let us denote $B_{i_{0}}^{\prime}:=50 K \lambda B_{i_{0}}$ (note that by $(i i)$ in the definition of the balls $B_{i}$, we have that $B_{i_{0}}^{\prime}$ is still contained in $\Omega_{2}$ ). Now, since for every $i \in I$ with $B_{i} \cap B_{i_{0}} \neq \emptyset$ the radius of $B_{i}$ is $C r_{i_{0}}$, we can estimate $\left|v_{B_{i}}-v_{10 B_{i_{0}}}\right|$ applying Lemma 29 again in the following way

$$
\begin{aligned}
\left|v_{B_{i}}-v_{10 B_{i_{0}}}\right| & \leq\left|v_{B_{i}}-u_{B_{i}}\right|+\left|u_{B_{i}}-u_{10 B_{i_{0}}}\right|+\left|u_{10 B_{i_{0}}}-v_{10 B_{i_{0}}}\right| \\
& \leq C r_{i_{0}}\left(f_{B_{i_{0}}^{\prime}} g^{Q} d z\right)^{\frac{1}{Q}} .
\end{aligned}
$$

Then,

$$
\begin{aligned}
\left|\sum_{i \in I}\left(v_{B_{i}}-v_{10 B_{i_{0}}}\right)\left(\theta_{i}(x)-\theta_{i}(y)\right)\right| & \leq \sum_{i \in I}\left|v_{B_{i}}-v_{10 B_{i_{0}}}\right|\left|\theta_{i}(x)-\theta_{i}(y)\right| \\
& \leq C d(x, y) \sum_{i \in I ; 2 B_{i} \cap B_{i_{0}} \neq \emptyset}\left|v_{B_{i}}-v_{10 B_{i_{0}}}\right| \frac{1}{r_{i}} \\
& \leq C d(x, y) \frac{1}{r_{i_{0}}} \sum_{i \in I ; 2 B_{i} \cap B_{i_{0}} \neq \emptyset}\left|v_{B_{i}}-v_{10 B_{i_{0}}}\right| \\
& \leq C d(x, y)\left(f_{B_{i_{0}}^{\prime}} g^{Q}\right)^{\frac{1}{Q}} .
\end{aligned}
$$

All together we have proved that

$$
|f(x)-f(y)| \leq|u(x)-u(y)|+C d(x, y)\left(f_{B_{i_{0}}^{\prime}} g^{Q}\right)^{\frac{1}{Q}}+\left|u(x)-u_{10 B_{i_{0}}}\right| .
$$

Now, as in the proof of Lemma 31 (the details this time are omitted), we deduce from (5.25) that

$$
G(x):=C g+C \sum_{i \in I ; x \in B_{i}}\left[\left(f_{B_{i_{0}}^{\prime}} g^{Q}\right)^{\frac{1}{Q}}+\left|u(x)-u_{B_{i}}\right|\right]
$$

is an upper gradient for $f$ in $A_{1}$. Now to finish the proof of the lemma, it suffices to control the $L^{Q}$-norm of $G$, which is the same computation as for (5.14) and (5.15) so we omit the proof here again. We get

$$
\|G\|_{L^{Q}\left(A_{1}\right)} \leq C \int_{W\left(A_{1}\right)} g^{Q} d \mu
$$

where $W\left(A_{1}\right):=A_{1} \cup \bigcup_{i \in I ; 2 B_{i} \cap A_{1} \neq \emptyset} 50 K \lambda B_{i}$. Since $W\left(A_{1}\right) \subset \Lambda_{\varepsilon}$ the proof of the lemma is complete for the case when $f$ is $K$-quasisymmetric. Now if $f$ is bi-Lipschitz 
all the above arguments work with $q \geq p$ instead of $Q$ which proves the lemma in its full generality.

We are now in a position to prove the following stability result.

Theorem 33. Let $(Q, K, p)$ be some given constants and let $f_{k}: \Omega_{k} \rightarrow \Omega$ be a sequence of $K$-quasisymmetric mappings into a fixed domain $\Omega \subseteq X$ satisfying $\mu(\partial \Omega)=0$ and such that $\left(X, d, \mu, f_{k}\right)$ satisfies the standard assumptions with constants $(Q, K, p)$. Assume in addition that

$$
\int_{\Omega} d\left(f_{k}^{-1}(x), x\right) d \mu \longrightarrow 0 \quad \text { and } \quad \Omega_{k} \stackrel{d_{H}^{c}}{\longrightarrow} \Omega
$$

Then $\mathscr{B}_{\Omega_{k}}^{Q} \rightarrow \mathscr{B}_{\Omega}^{Q}$ in the sense of Mosco. In addition, if the sequence of functions $f_{k}$ are bi-Lipschitz (with same constants) then $\mathscr{B}_{\Omega_{k}}^{q} \rightarrow \mathscr{B}_{\Omega}^{q}$ for all $q \geq p$.

Proof. We begin by proving condition (M1). Let $u \in N^{1, Q}(\Omega)$ and for $k \in \mathbf{N}$ big enough we define $u_{k}:=\tilde{u}_{k}$ the function given by Lemma 32 with $\Omega$ and $\Omega_{k}$. We already know that $u_{k} \in N^{1, Q}\left(\Omega_{k}\right)$. Therefore, all we have to prove is that $\left(u_{k} \chi_{\Omega_{k}}, D u_{k} \chi_{\Omega_{k}}\right)$ converges strongly to $\left(u \chi_{\Omega}, D u \chi_{\Omega}\right)$ in $L^{Q}(X) \times L^{Q}\left(X, \mathbf{R}^{N}\right)$.

We denote

$$
\Lambda_{\varepsilon}:=\left\{y \in \Omega ; d(y, \partial \Omega) \leq c \varepsilon^{\frac{1}{1+Q}}\right\},
$$

and we also define

$$
\varepsilon_{k}=\int_{\Omega} d\left(x, f_{k}^{-1}(x)\right) d \mu(x)+200 \lambda K d_{H}\left(\Omega_{k}^{c}, \Omega^{c}\right) \quad \text { and } \quad \Lambda_{k}:=f_{k}^{-1}\left(\Lambda_{\varepsilon_{k}}\right) .
$$

Let us first prove that

$$
\mu\left(\Lambda_{k}\right) \rightarrow 0 \text { when } k \rightarrow+\infty .
$$

Indeed, let $\eta$ be any small constant. For $k$ big enough we have that

$$
\int_{\Omega} d\left(x, f_{k}^{-1}(x)\right) d \mu(x) \leq \eta
$$

and so, if we denote

$$
A_{\eta}:=\left\{y \in \Omega ; d\left(y, f_{k}^{-1}(y)\right) \geq \sqrt{\eta}\right\},
$$

we obtain by applying Tchebychev's inequality that

$$
\mu\left(A_{\eta}\right) \leq \frac{1}{\sqrt{\eta}} \int_{\Omega} d\left(x, f_{k}^{-1}(x)\right) d \mu(x) \leq \sqrt{\eta} .
$$

Now observe that

Therefore,

$$
f_{k}^{-1}\left(\Lambda_{\varepsilon_{k}}\right) \backslash A_{\eta} \subseteq \Lambda_{\varepsilon_{k}+\sqrt{\eta}} .
$$

$$
\begin{aligned}
\mu\left(f_{k}^{-1}\left(\Lambda_{\varepsilon_{k}}\right)\right) & \leq \mu\left(f_{k}^{-1}\left(\Lambda_{\varepsilon_{k}}\right) \cap A_{\eta}\right)+\mu\left(f_{k}^{-1}\left(\Lambda_{\varepsilon_{k}}\right) \backslash A_{\eta}\right) \\
& \leq \mu\left(A_{\eta}\right)+\mu\left(\Lambda_{\varepsilon_{k}+\sqrt{\eta}}\right) \leq \sqrt{\eta}+\mu\left(\Lambda_{\varepsilon_{k}+\sqrt{\eta}}\right),
\end{aligned}
$$

which implies

$$
0 \leq \limsup _{k \rightarrow+\infty} \mu\left(\Lambda_{k}\right) \leq \sqrt{\eta}+\mu\left(\Lambda_{\sqrt{\eta}}\right) .
$$

Since $\eta$ is arbitrary, if we let $\eta \rightarrow 0$ in (5.28), we deduce (5.27). 
Now applying Lemma 32 we can write

$$
\left(\int_{X}\left|u_{k} \chi_{\Omega_{k}}-u \chi_{\Omega}\right|^{Q} d \mu\right)^{\frac{1}{Q}}=\left(\int_{\Lambda_{k}}\left|u_{k} \chi_{\Omega_{k}}-u \chi_{\Omega}\right|^{Q} d \mu\right)^{\frac{1}{Q}} \leq C\|u\|_{L^{Q}\left(\Lambda_{k}\right)},
$$

which tends to zero when $k \rightarrow+\infty$ because of (5.27). For the gradients, a similar argument can be done, that is,

$$
\left(\int_{X}\left|D u_{k} \chi_{\Omega_{k}}-D u \chi_{\Omega}\right|^{Q} d \mu\right)^{\frac{1}{Q}}=\left(\int_{\Lambda_{k}}\left|D u_{k} \chi_{\Omega_{k}}-D u \chi_{\Omega}\right|^{Q} d \mu\right)^{\frac{1}{Q}} \leq C\|D u\|_{L^{Q}\left(\Lambda_{k}\right)},
$$

which tends to 0 as $k$ tends to infinity. Thus (M1) is proved.

Let us now prove (M2). Let $h_{k}$ be a sequence of indices converging to $\infty, u_{k}$ is a sequence such that $u_{k} \in N^{1, Q}\left(\Omega_{h_{k}}\right)$ for every $k$, and $u_{k} \chi_{\Omega_{h_{k}}}$ converges weakly in $L^{Q}(X)$ to a function $\phi$, while $D u_{k} \chi_{\Omega_{h_{k}}}$ converges weakly in $L^{Q}\left(X, \mathbf{R}^{N}\right)$ to a function $\psi$. Let $\varphi \in L^{Q}(X)$ be compactly supported in $\Omega$. By the weak convergence we have that

$$
\left\{\begin{array}{l}
\int_{\Omega} u_{k} \chi_{\Omega_{h_{k}}} \varphi d \mu \stackrel{k \rightarrow+\infty}{\longrightarrow} \int_{\Omega} \phi \varphi d \mu, \\
\int_{\Omega}\left\langle D u_{k} \chi_{\Omega_{h_{k}}}, \varphi\right\rangle d \mu \stackrel{k \rightarrow+\infty}{\longrightarrow} \int_{\Omega} \psi \varphi d \mu .
\end{array}\right.
$$

On the other hand, since $\Omega_{h_{k}}$ converges to $\Omega$ for the complementary Hausdorff distance, for $k$ large enough the function $\chi_{\Omega_{h_{k}}}$ is equal to 1 everywhere on the support of $\phi$. Thus (5.29) shows that $u_{k}$ converges to $\phi$ weakly in $L^{Q}(\Omega)$ and $D u_{k}$ converges to $\psi$ weakly in $L^{Q}(\Omega)$. By Lemma 6 we conclude that $\psi$ is an upper gradient for $\phi$. Moreover since $\psi \in L^{Q}\left(X, \mathbf{R}^{N}\right)$, we deduce that $\left.\phi\right|_{\Omega} \in N^{1, Q}(\Omega)$. To conclude, all we have to show is that $\varphi=\psi=0$ in $\Omega^{c}$. To see this, we use a similar argument as above by defining a function $\varphi$ compactly supported in $\Omega^{c}$. By the weak convergence, and because $\Omega_{k}$ converges to $\Omega$ for the complementary Hausdorff distance, we deduce that $\int_{\Omega} \phi \varphi d x=0$. This holds for any function $\varphi$ compactly supported in $\Omega^{c}$. Since $\phi \in L^{Q}(X)$ we conclude that $\phi=0$ a.e. in $\Omega^{c}$. In a similar way we obtain that $\psi=0$ in $\Omega^{c}$ and since $\mu(\partial \Omega)=0$ we have that $\phi=\phi \chi_{\Omega}$ and $\psi=\psi \chi_{\Omega} \mu$-a.e. All together we have proved that $\mathscr{B}_{\Omega_{k}}^{Q}$ converges to $\mathscr{B}_{\Omega}^{Q}$ in the sense of Mosco.

If $f_{k}$ are bi-Lipschitz the proof works in the same way replacing $Q$ by $q \geq p$ and the proof of the theorem is now complete.

Remark 34. Notice that in the proof of Theorem 33 we only used that $f_{k}^{-1}$ converges to the identity map in measure so that we could slightly weaken the assumption $\int_{\Omega} d\left(f_{k}^{-1}(x), x\right) d \mu \rightarrow 0$ if necessary.

5.1. An example. We would like to emphasize the fact that for some sequences of domains, Theorem 33 applies whereas Theorem 16 not. For instance, let us define

$$
\Omega_{t}:=\left\{(x, y) \in \mathbf{R}^{2} ;|x| \leq 1 \text { and } 0<y<(2-\sqrt{|x|}) t\right\} \text {. }
$$

Observe that for $t \in(0,1), \Omega_{t}$ is never an extension domain for $W^{1,2}\left(\Omega_{t}\right)$ thus we cannot apply Theorem 16 to prove some $\gamma$-convergence results for $\Omega_{t}$. On the other 
hand for a fixed $t_{0}>0$, one can consider the bi-Lipschitz mappings $g_{t}: \Omega_{t} \rightarrow \Omega_{t_{0}}$ defined by

$$
g_{t}:(x, y) \mapsto\left(x, \frac{t_{0}}{t} y\right)
$$

It is easily seen that

$$
\int_{\Omega} d\left(x, g_{t}^{-1}(x)\right) d x \leq \mathscr{L}^{2}\left(\Omega_{t}\right)\left|1-\frac{t_{0}}{t}\right| \stackrel{t \rightarrow t_{0}}{\longrightarrow} 0 .
$$

Therefore, by applying Theorem 33, the $\gamma$-convergence of $\Omega_{t}$ to $\Omega_{t_{0}}$ holds when $t \rightarrow t_{0}$.

5.2. Application for shape optimisation problems. In this last subsection we use Theorem 33 to prove an existence result for a class of shape optimisation problems (with Neumann boundary conditions) under quasiconformal deformations. We found it more concrete to fit this application in $\mathbf{R}^{N}$, but one could get a similar result in more general metric spaces without substantial changes.

We say that $\Omega_{k} \gamma_{p}$-converges to $\Omega$ if $\mathscr{B}_{\Omega_{k}}^{p} \rightarrow \mathscr{B}_{\Omega}^{p}$ in the sense of Mosco. Let $\mathscr{A}$ be a class of domains in $\mathbf{R}^{N}$, and let $F$ be a functional defined on $\mathscr{A}$. We say that $F$ is lower-semicontinuous with respect to $\gamma_{p}$-convergence if

$$
F(\Omega) \leq \liminf _{k \rightarrow+\infty} F\left(\Omega_{k}\right)
$$

whenever $\Omega_{k}$ is a sequence of domains that $\gamma_{p}$-converges to $\Omega$. A classical example is the eigenvalue problem. If $F(\Omega):=\lambda_{1}(\Omega)$ where $\lambda_{1}$ is the first eigenvalue of the Laplacian in $\Omega$ (with Neumann boundary conditions), then it is well known that $\lambda_{1}(\Omega)$ is lower semicontinuous with respect to $\gamma_{2}$-convergence. We refer to $[5,3,18]$ for other examples of lower-semicontinuous functionals.

Let $\Omega_{0} \subset \mathbf{R}^{N}$ be a fixed bounded domain. We denote by $\mathscr{A}\left(\Omega_{0}\right)$ the family of quasiconformal maps $g: \Omega_{0} \rightarrow g\left(\Omega_{0}\right)$ satisfying:

(i) $g\left(\Omega_{0}\right)$ is contained in a fixed ball,

(ii) $g$ lies in $W^{1, p}$ with norm less than $C$ and with $p>N$, and

(iii) the distortion constant of $g$ is less than $K$.

A consequence of our stability result is the following existence theorem for minimizers of such functionals.

Theorem 35. For any lower-semicontinuous functional $F$ with respect to $\gamma_{N^{-}}$ convergence, there exists a minimizer for the problem

$$
\min _{g \in \mathscr{A}\left(\Omega_{0}\right)} F\left(g\left(\Omega_{0}\right)\right) .
$$

Proof. Let $\Omega_{k}:=g_{k}\left(\Omega_{0}\right)$ be a minimizing sequence for the problem (5.30). Since $\Omega_{k}$ is contained in a fixed ball, after passing to a subsequence if necessary we may assume that there exists a domain $\Omega$ such that $d_{H}\left(\Omega_{k}^{c}, \Omega^{c}\right) \rightarrow 0$. On the other hand, since the functions $g_{k}$ are equibounded in $W^{1, p}$ with $p>N$, by the Sobolev embedding they are equicontinuous and equibounded in $L^{\infty}$. Therefore, one can extract a subsequence of $g_{k}$ that converges uniformly on every compact subset of $\Omega_{0}$ to a function $g$. By classical results on quasiconformal maps (see [28]), we deduce that the limit $g$ is a $K$-quasiconformal homeomorphism. This allows us to consider $g_{k} \circ g^{-1}$ that converges in $L^{1}$ to Id on $\Omega$. Indeed, let $\varepsilon>0$ and let us choose a 
compact set $K \subset \Omega_{0}$ with $\mathscr{L}^{N}\left(g\left(\Omega_{0} \backslash K\right)\right) \leq \varepsilon$. We have that

$$
\begin{aligned}
& \int_{g\left(\Omega_{0}\right)}\left\|g_{k} \circ g^{-1}(x)-x\right\| d \mathscr{L}^{N}=\int_{\Omega_{0}}\left\|g_{k}(x)-g(x)\right\| J_{g}(x) d \mathscr{L}^{N} \\
& =\int_{\Omega_{0} \backslash K}\left\|g_{k}(x)-g(x)\right\| J_{g}(x) d \mathscr{L}^{N}+\int_{K}\left\|g_{k}(x)-g(x)\right\| J_{g}(x) d \mathscr{L}^{N} .
\end{aligned}
$$

Since $g_{k}$ converges uniformly to $g$ on every compact subset of $\Omega_{0}$, we get that

$$
\int_{K}\left\|g_{k}(x)-g(x)\right\| J_{g}(x) d \mathscr{L}^{N} \leq\left\|g-g_{k}\right\|_{\infty} \mathscr{L}^{N}(\Omega) \longrightarrow 0 \text { as } k \rightarrow \infty .
$$

Moreover, due to the fact that $\mathscr{L}^{N}\left(g\left(\Omega_{0} \backslash K\right)\right) \leq \varepsilon$ and $\left\|g_{k}(x)-g(x)\right\| \leq C$,

$$
\int_{\Omega_{0} \backslash K}\left\|g_{k}(x)-g(x)\right\| J_{g}(x) d \mathscr{L}^{N} \leq C \varepsilon .
$$

Since $\varepsilon$ was arbitrary, all together we obtain that

$$
\int_{g\left(\Omega_{0}\right)}\left\|g_{k} \circ g^{-1}(x)-x\right\| d \mathscr{L}^{N} \longrightarrow 0 \text { as } k \rightarrow \infty
$$

Then applying Theorem 33 we deduce that $\mathscr{B}_{\Omega_{k}}^{N} \rightarrow \mathscr{B}_{\Omega}^{N}$ in the sense of Mosco. And since

$$
F(\Omega) \leq \liminf _{k \rightarrow+\infty} F\left(\Omega_{k}\right)
$$

we conclude that $\Omega$ is a minimizer for the problem (5.30).

Remark 36. A similar result could be obtained with bi-Lipschitz mappings instead of quasiconformal mappings replacing $\gamma_{N^{-}}$convergence assumption by $\gamma_{p^{-}}$ convergence with any $p \geq 1$.

Remark 37. The same proof would also give a similar result replacing condition (ii) in the definition of the family $\mathscr{A}\left(\Omega_{0}\right)$ by any assumption that would imply the equicontinuity of the family $\mathscr{A}\left(\Omega_{0}\right)$ in order to get compactness and closure of the considered family of quasiconformal mappings.

\section{References}

[1] Buörn, A., J. Buörn, and N. Shanmugalingam: The Dirichlet problem for $p$-harmonic functions on metric spaces. - J. Reine Angew. Math. 556, 2003, 173-203.

[2] Björn, J., and N. Shanmugalingam: Poincaré inequalities, uniform domains and extension properties for Newton-Sobolev functions in metric spaces. - J. Math. Anal. Appl. 332:1, 2007, 190-208.

[3] Bucur, D., and G. Buttazzo: Variational methods in shape optimization problems. - Progr. Nonlinear Differential Equations Appl. 65, Birkhäuser Boston Inc., Boston, MA, 2005.

[4] Bucur, D., and N. Varchon: Boundary variation for a Neumann problem. - Ann. Scuola Norm. Sup. Pisa Cl. Sci. (4) 29:4, 2000, 807-821.

[5] Buttazzo, G., and G. Dal Maso: An existence result for a class of shape optimization problems. - Arch. Rational Mech. Anal. 122:2, 1993, 183-195.

[6] Chambolle, A., and F. Doveri: Continuity of Neumann linear elliptic problems on varying two-dimensional bounded open sets. - Comm. Partial Differential Equations 22:5-6, 1997, 811840 . 
[7] Chemger, J.: Differentiability of Lipschitz functions on metric measure spaces. - Geom. Funct. Anal. 9:3, 1999, 428-517.

[8] Chenais, D.: On the existence of a solution in a domain identification problem. - J. Math. Anal. Appl. 52:2, 1975, 189-219.

[9] Dal Maso, G.: An introduction to $\Gamma$-convergence. - Progr. Nonlinear Differential Equations Appl. 8, Birkhäuser Boston Inc., Boston, MA, 1993.

[10] Dal Maso, G., F. Ebobisse, and M. Ponsiglione: A stability result for nonlinear Neumann problems under boundary variations. - J. Math. Pures Appl. (9) 82:5, 2003, 503-532.

[11] Del Vecchio, T.: The thick Neumann's sieve. - Ann. Mat. Pura Appl. (4) 147, 1987, 363-402.

[12] Franchi, B., P. HajŁasz, and P. Koskela: Definitions of Sobolev classes on metric spaces. - Ann. Inst. Fourier (Grenoble) 49:6, 1999, 1903-1924.

[13] HajŁasz, P.: Sobolev spaces on metric-measure spaces. - In: Heat kernels and analysis on manifolds, graphs, and metric spaces (Paris, 2002), Contemp. Math. 338, 173-218.

[14] HajŁasz, P., and P. Koskela: Sobolev met Poincaré. - Mem. Amer. Math. Soc. 145:688, 2000.

[15] Heinonen, J. and P. Koskela: From local to global in quasiconformal structures. - Proc. Nat. Acad. Sci. U.S.A. 93:2, 1996, 554-556.

[16] Heinonen, J. and P. Koskela: Quasiconformal maps in metric spaces with controlled geometry. - Acta Math. 181:1, 1998, 1-61.

[17] Heinonen, J., P. Koskela, N. Shanmugalingam, and J. T. Tyson: Sobolev classes of Banach space-valued functions and quasiconformal mappings. - J. Anal. Math. 85, 2001, 87139.

[18] Henrot, A., and M. Pierre: Variation et optimisation de formes, une analyse géométrique, mathématiques et applications. - Springer, 2005.

[19] Jones, P. W.: Quasiconformal mappings and extendability of functions in Sobolev spaces. Acta Math. 147:1-2, 1981, 71-88.

[20] Keith, S., and X. Zhong: The Poincaré inequality is an open ended condition. - Ann. of Math. (2) 167:2, 2008, 575-599.

[21] Koskela, P., and P. Macmanus: Quasiconformal mappings and Sobolev spaces. - Studia Math. 131:1, 1998, 1-17.

[22] Koskela, P., N. Shanmugalingam , and J. T. Tyson: Dirichlet forms, Poincaré inequalities, and the Sobolev spaces of Korevaar and Schoen. - Potential Anal. 21:3, 2004, 241-262.

[23] Lemenant, A., and E. Milakis: A stability result for nonlinear Neumann problems in Reifenberg flat domains in $\mathbf{R}^{N}$. - Preprint, available on CVGMT.

[24] Lemenant, A., and E. Milakis: Quantitative stability for the first Dirichlet eigenvalue in Reifenberg flat domains in $\mathbf{R}^{N}$. - J. Math. Anal. Appl. 364:2, 2010, 522-533.

[25] Mosco, U.: Convergence of convex sets and of solutions of variational inequalities. - Advances in Math. 3, 1969, 510-585.

[26] Murat, F.: The Neumann sieve. - In: Nonlinear variational problems (Isola d'Elba, 1983), Res. Notes in Math. 127, 1985, Pitman, Boston, MA, 24-32.

[27] Shanmugalingam, N.: Newtonian spaces: an extension of Sobolev spaces to metric measure spaces. - Rev. Mat. Iberoamericana 16:2, 2000, 243-279.

[28] VÄIsÄLÄ, J.: Lectures on n-dimensional quasiconformal mappings. - Lecture Notes in Math. 229, Springer-Verlag, Berlin, 1971.

[29] VÄIsÄlÄ, J.: Quasiconformal maps of cylindrical domains. - Acta Math. 162:3-4, 1989, 201225.

Received 30 September 2009 